DOI: 10.1002/adsc.200((will be filled in by the editorial sttaff))

\title{
Regioselective Synthesis of Heteroatom-Functionalized Cyclobutene-triflones and Cyclobutenones
}

\author{
Benito Alcaide, ${ }^{\mathrm{a} *}$ Pedro Almendros, ${ }^{\mathrm{b} *}$ and Carlos Lázaro-Milla ${ }^{\mathrm{a}}$
}

a Grupo de Lactamas y Heterociclos Bioactivos, Departamento de Química Orgánica I, Unidad Asociada al CSIC, Facultad de Química, Universidad Complutense de Madrid, 28040 Madrid, Spain, Fax: (+34) 91-3944103, E-mail: alcaideb@quim.ucm.es

b Instituto de Química Orgánica General, Consejo Superior de Investigaciones Científicas, IQOG-CSIC, Juan de la Cierva 3, 28006 Madrid, Spain, Fax: (+34) 91-5644853, E-mail: Palmendros@iqog.csic.es

Received: ((will be filled in by the editorial staff))

Supporting information for this article is available on the WWW under http://dx.doi.org/10.1002/adsc.200\#\#\#\#\#.((Please delete if not appropriate))

\begin{abstract}
The controlled metal-free synthesis of a vast variety of heteroatom-containing cyclobutene-triflones and cyclobutenones has been developed starting from heteroatomsubstituted alkynes and a pyridinium salt (a latent $\mathrm{Tf}_{2} \mathrm{C}=\mathrm{CH}_{2}$

reactions allows for the selective preparation of oxygen-, nitrogen-, bromine-, chlorine-, iodine-, sulphur-, selenium-, tellurium-, phosphorus-, and silicon-functionalized cyclobutene derivatives.
\end{abstract} source). This powerful methodology, involving cyclization
Keywords: alkynes; annulation; carbocycles; fluorine; synthetic methods

\section{Introduction}

The importance of the synthesis of the cyclobutene core is ever increasing in relation to its presence in natural products and biologically active substances. ${ }^{[1]}$ In addition to its biological importance, this strained carbocycle serves as versatile building block and has attracted considerable attention in organic synthesis. ${ }^{[2]}$ One of the most challenging issues in synthesizing cyclobutenes is how to efficiently and selectivity introduce functionality into the four-membered carbocyclic skeleton. We have recently communicated the cyclization reaction of alkynes and $\mathrm{Tf}_{2} \mathrm{C}=\mathrm{CH}_{2}$ to afford 1-aryl-2-alkyl-4,4-bis(triflyl)cyclobutenes, but this method was restricted so far to 1-aryl-2alkyl(aryl)substituted alkynes. ${ }^{[3]}$ Due to the marked influence on the physical, chemical, and biological properties of small molecules imparted by the presence of heteroatoms, we envisaged the development of a mild method for the preparation of cyclobutene derivatives bearing an extra heteroatom directly linked to a $\mathrm{sp}^{2}$ carbon atom of the carbocycle (Scheme 1). These heteroatom-substituted cyclobutenetriflones. ${ }^{[4],[5]}$ should bring together the new properties conferred by heteroatoms and trifyl group with the exceptionally rich chemistry of cyclobutenes.
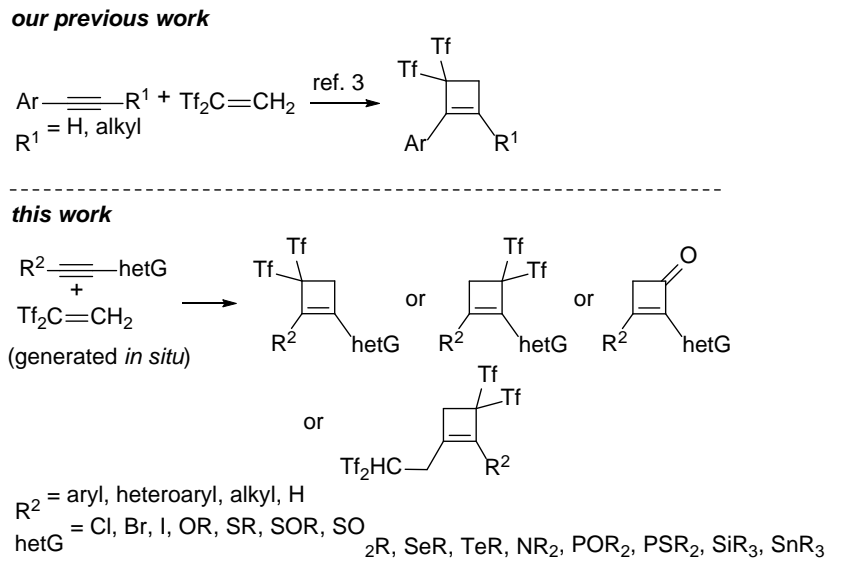

Scheme 1. Metal-free room temperature synthesis of heteroatom-substituted cyclobutene-triflones or cyclobutenones.

\section{Results and Discussion}

To explore the effect of various heteroatomic substituents on cyclobutene-triflone formation, a number of differently functionalized alkynes were selected. 1-Chloroalkyne 1a was chosen as model substrate to optimize suitable conditions for the reaction with pyridinium salt 2 , a $\mathrm{Tf}_{2} \mathrm{C}=\mathrm{CH}_{2}$ source. Zwitterion $\mathbf{2}$ is poorly soluble in apolar or halogenated solvents, which limited the optimization of the solvent 
parameter. Acetonitrile at room temperature was identified as the best choice for the reaction of 1chloroalkyne 1a with $\mathbf{2}$. Notably, the reaction of 1a with 2 led to bis(trifluoromethylsulfonyl)chlorocyclobutene $3 a$, which was obtained in good yield (84\%) as single regioisomer without the requirement of any catalyst (Scheme 2). Addition of $\mathrm{H}_{2} \mathrm{O}$ may be beneficial by enhancing the solubility of the zwitterionic reagent 2. However, when the reaction was carried out in a mixture acetonitrile/water (1:1), chlorocyclobutene 3a was obtained in decreased yield (70\%). We further investigated the effect of different halogens on the cyclization as shown in Scheme 2. Reaction of 1bromo(iodo)alkynes 1a-f with $\mathrm{Tf}_{2} \mathrm{C}=\mathrm{CH}_{2}$ afforded cycloadducts $\mathbf{3 b}-\mathbf{f}$ as sole products in $73-97 \%$ yields. Electron-rich substituents accelerated the reaction progress, as exemplified with the formation of bromocyclobutene-triflone $\mathbf{3 f}$ in just 10 minutes. The structure and regiochemistry of compound $\mathbf{3 d}$ was unambiguously assigned through its X-ray crystallographic analysis (Figure 1). ${ }^{[6]}$

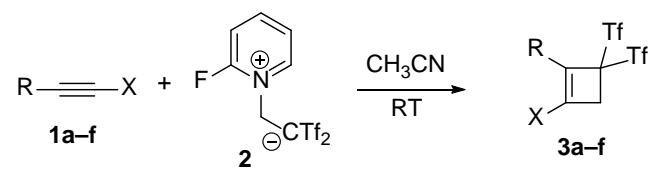

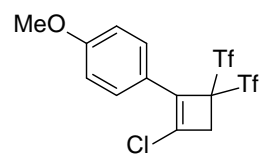

$\left.3 \mathbf{a}_{(84 \%, 3} \mathrm{h}\right)$

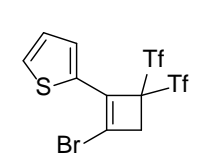

3d $(85 \%, 2$ h)

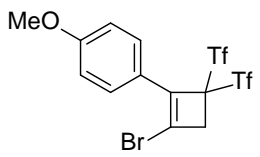

3b $(92 \%, 3$ h)

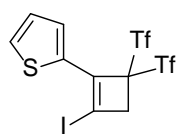

3e $(96 \%, 2$ h)

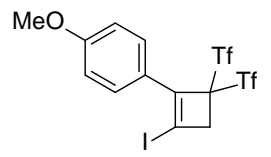

3c $(97 \%, 3 \mathrm{~h})$

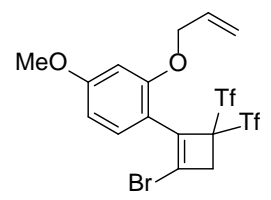

$3 \mathbf{f}(73 \%, 10 \mathrm{~min})$
Scheme 2. Controlled preparation of bis(trifluoromethylsulfonyl) halocyclobutenes 3.

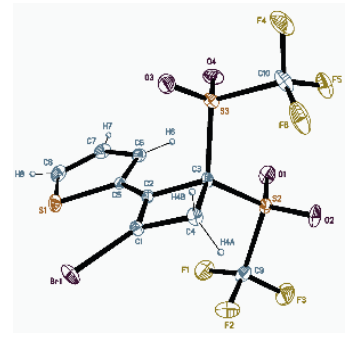

Figure $1 . \quad$ ORTEP drawing of bis(trifluoromethylsulfonyl)bromocyclobutene 3d. Thermal ellipsoids shown at $50 \%$ probability.

With the best halocyclobutene-triflone formation conditions identified, the scope of this transformation was then examined in alkynyl-ethers, -thioethers, selenoethers, and -teluroethers 4 . Cyclizations adducts 5a-d could not be obtained at RT in reasonable yield because ynol ethers $\mathbf{4 a - d}$ quickly reacted in contact with zwitterion 2, resulting in a complicated reaction. Fortunately, the reactions were more effective at $0{ }^{\circ} \mathrm{C}$, and ynol ethers 4a-d undergo smooth cyclization to afford cyclic enol ethers $\mathbf{5 a - d}$ in reasonable yields (Scheme 3). Remarkably, the presence of OR groups instead halogens at the starting alkyne reversed the product distribution completely, implying that the choice of substituents can control the regioselectivity of the reaction.

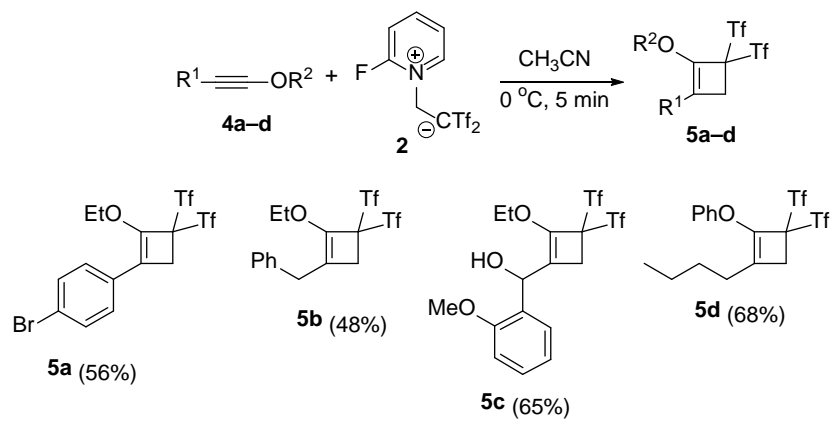

Scheme 3. Controlled preparation of bis(trifluoromethylsulfonyl) enol ether cyclobutenes 5a-d.

Organosulfur compounds occupy a special position in heteroatom-containing small molecules, both as bioactive compounds as well as synthetic intermediates. ${ }^{[7]}$ We envisaged the preparation of cyclobutene-triflones bearing S-based groups starting from thia-alkynes. The proposed thia-cyclobutenetriflones present intringuing structures, which stability was initially of question given the supposed instability of chalcogen substituted cyclobutene-triflones. Pleasingly, when using thia-alkynes $\mathbf{4 e - \mathbf { j }}$ and pyridinium salt $\mathbf{2}$, thia-cyclobutene-triflones $\mathbf{5 e}-\mathbf{j}$ were isolated in high yields (Scheme 4), indicating the feasibility of this type of structures. In the case of sulphur-based substrates, the same level and sense of regioselectivity as in oxygen derivatives was observed.

Cyclization precursors $\mathbf{4 k - p}$ bearing Se and Te carbon chains were prepared. The standard cyclobutene formation conditions were then applied across this range of substrates. Alkynes $4 \mathbf{k}-\mathbf{p}$ efficiently formed the desired polyfunctionalized fourmembered rings $\mathbf{5 k}-\mathbf{p},{ }^{[8]}$ but the cyclization proved capricious for Se-derivative $5 \mathbf{l}$ due to partial degradation of the final material under the chromatographic purification conditions (Scheme 4). Interestingly, in all cases the product exhibited the same regiocontrol of oxygen and sulphur derivatives. 


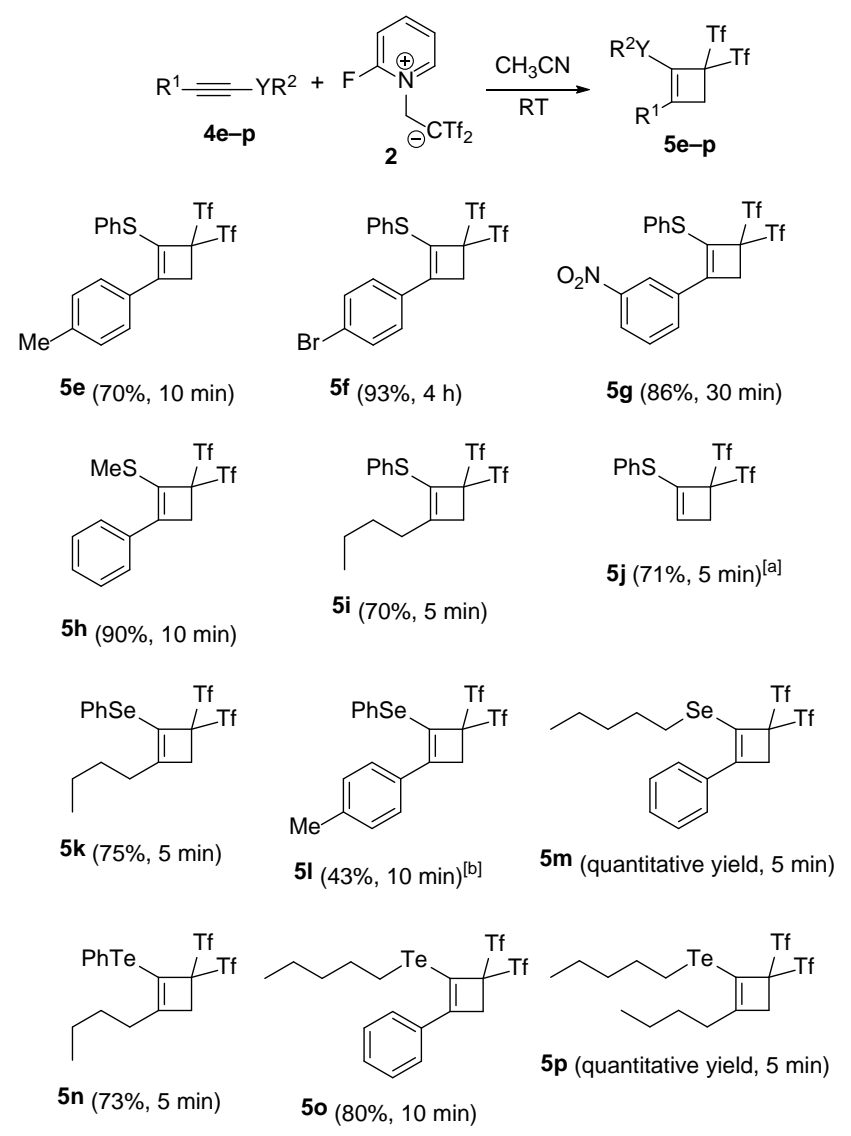

Scheme 4. Controlled preparation of bis(trifluoromethylsulfonyl) chalcogen cyclobutenes 5. [a] The reaction was carried out at $0{ }^{\circ} \mathrm{C}$. [b] Partial decomposition during chromatographic purification.

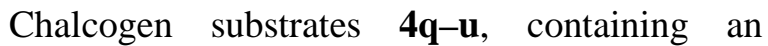
additional electron-rich substituent at the other alkyne side, worked under the standard conditions to afford unexpected cyclobutene derivatives along with unidentified by-products. Cyclobutenones 6q-u can be accessed from this type of substrates albeit with a lower yield (Scheme 5).
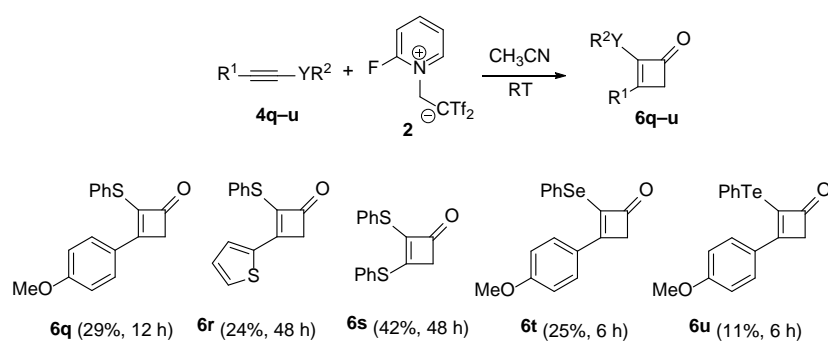

Scheme 5. Preparation of bis(trifluoromethylsulfonyl) chalcogen cyclobutenones $\mathbf{6}$.

The influence of different sulphur oxidation states on the reactivity was also tested. In addition of thiaalkynes $\mathbf{4 e - j}$ we were interested in examining sulfinyl alkynes 8a-h and sulfonyl alkyne 8i. Firstly, the electronic effect on the S-substituent was investigated and the result showed that substrates bearing aliphatic substituents (8a) or deactivated benzene rings (8b) did not afford the desired cyclobutenes. By contrast, neutral or electron-rich aromatic substituents can be tolerated to provide sulfinyl cyclobutenes $\mathbf{9 c - h}$ and sulfonyl cyclobutene 9i (Scheme 6). Notably, a regiochemistry reversal was observed from sulphur with oxidation number -2 such as in alkynes $\mathbf{4 e - j}$ and sulphur with oxidation numbers +4 (alkynes $\mathbf{8 c}-\mathbf{h}$ ) and +6 (alkyne 8i).

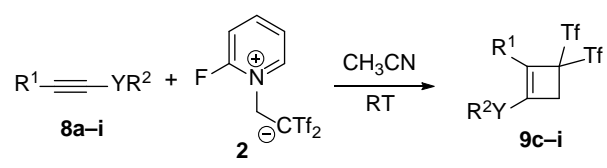

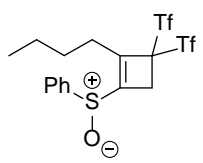

$9 a(0 \%, 24 \mathrm{~h})$

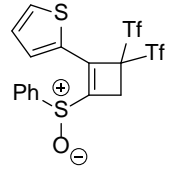

9f $(53 \%, 10 \mathrm{~min})$

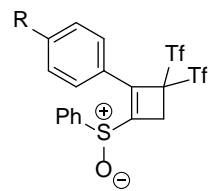

9b $(\mathrm{R}=\mathrm{Br}, 0 \%, 24 \mathrm{~h})$ 9c $(R=M e, 46 \%, 10 \mathrm{~min})$ 9d $(\mathrm{R}=\mathrm{MeO}, 51 \%, 10 \mathrm{~min})$

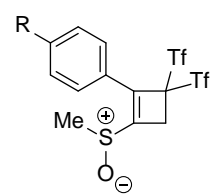

$9 \mathbf{g}(\mathrm{R}=\mathrm{H}, 47 \%, 10 \mathrm{~min})$ 9h $(R=$ MeO, 57\%, $10 \mathrm{~min})$

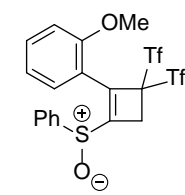

$9 \mathbf{e}(31 \%, 10 \mathrm{~min})$

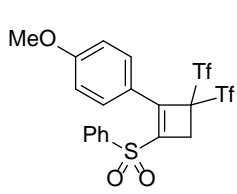

9i (quantitative yield, $12 \mathrm{~h}$ )
Scheme 6. Controlled preparation of bis(trifluoromethylsulfonyl) sulfinyl cyclobutenes $\mathbf{9 d - h}$ and sulfonyl cyclobutene $\mathbf{9 i}$.

The effect of an amino group in the fourmembered ring formation reaction was investigated with the use of indole-based ynamine derivatives 10al. As in the case of OR, SR, SeR, and TeR substituents (Schemes 3-5), the regioselectivity reversal is dictated by the heteroatom. Ynamines 10a-l afforded the corresponding cyclobutenes $\mathbf{1 1 a}-\mathbf{l}$ as the sole products in fair yields (Scheme 7). These examples also indicated that ynamines $11 \mathbf{a}, \mathbf{1 1 h}$, and $11 \mathbf{i}$ bearing the simple phenyl ring at $\mathrm{N} 1$ are the best starting materials, furnishing the highest yields (quantitative or almost quantitative yields) of the appropriate cyclobutenes. Unexpectedly, results in Scheme 8 show, in all instances, that ynamines $\mathbf{1 0} \mathbf{m}-\mathbf{t}$ having electron-rich $4 \mathrm{MeOC}_{6} \mathrm{H}_{4}$ groups furnished exclusively

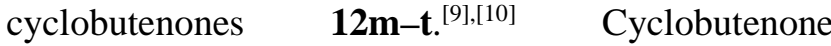
formation is spontaneous under the reaction conditions, but it may be facilitated by the addition of water ( 2 equiv) and $\mathrm{K}_{2} \mathrm{CO}_{3}$ (2 equiv) during the work-up. 


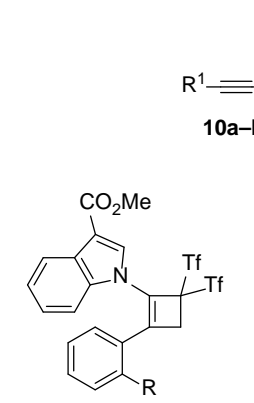

11a $\mathrm{R}=\mathrm{H}(94 \%, 4 \mathrm{~h})$ 11b $\mathrm{R}=\mathrm{MeO}(33 \%, 3 \mathrm{~h})$
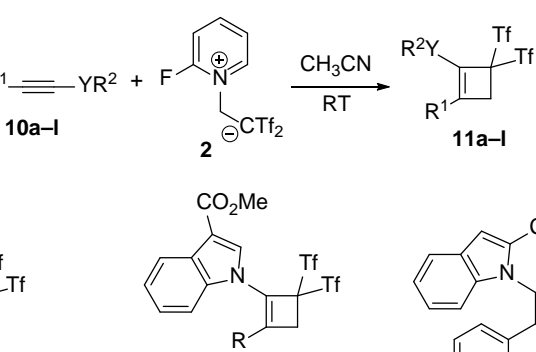

11c $\mathrm{R}=\mathrm{H}(67 \%, 1 \mathrm{~min})[\mathrm{a}]$ 11d $\mathrm{R}=2$-thienyl $\left(29 \% \frac{1}{1} \mathrm{~h}\right)$, h) $\quad \mathbf{1 1 h}$ (quantitative $11 \mathrm{e} \mathrm{R}=3-\mathrm{MeOC}_{6} \mathrm{H}_{4}(82 \%, 5 \mathrm{~h}) \quad$ yield, $\left.1 \mathrm{~h}\right)$ $11 f \mathrm{R}=3-\mathrm{NO}_{2} \mathrm{C}_{6} \mathrm{H}_{4}$

11g $\mathrm{R}=n-\mathrm{Bu}(84 \%, 5 \mathrm{~min})$

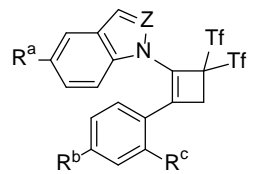

11i $\mathrm{R}^{\mathrm{a}}=\mathrm{H}, \mathrm{R}_{\mathrm{b}}=\mathrm{H}, \mathrm{R}_{\mathrm{c}}=\mathrm{H}, \mathrm{Z}=\mathrm{N}$ (quantitative yield, $3 \mathrm{~h}$ )

11i $R^{a}=H, R_{b}=H, R_{c}=M e O, Z=N(40 \%, 1 h)$

11j $R^{a}=B r, R_{b}=B r, R_{c}=H, Z=C H(72 \%, 30 \mathrm{~min})$

Scheme 7. Controlled preparation of bis(trifluoromethylsulfonyl) indolyl cyclobutenes 11a-l. [a] The reaction was carried out at $0{ }^{\circ} \mathrm{C}$.

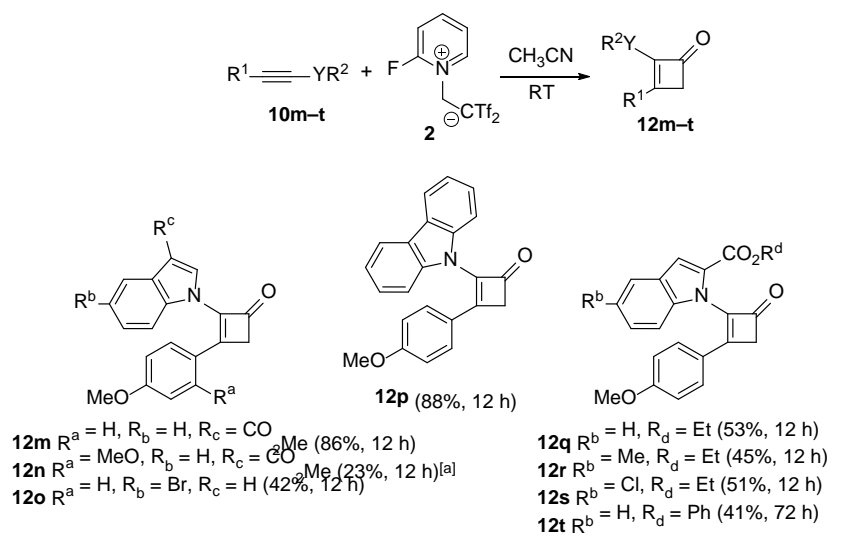

Scheme 8. Controlled preparation of bis(trifluoromethylsulfonyl) indolyl cyclobutenones 12m-t. [a] Messy reaction.

As shown in Scheme 9, the mechanism for the cyclobutenone formation involves two main processes, namely, cyclobutene ring construction and hydrolysis. The proposal for the first process (formation of 11mt) is based on our previous DFT studies of 1-aryl-2alkyl-4,4-bis(triflyl)cyclobutenes, ${ }^{[5]}$ but now the regioselectivity is dictated by the electronic effects of the heterocyclic amine. Adventitious water in the reaction medium is required for the double trifluoro(hydrosulfonyl)methane (TfH) elimination, giving rise to hydrates $\mathbf{1 7}$. This twofold water addition is assisted by the resonance effect of the 4-methoxy substituent in the 4-methoxyphenyl group. Finally, dehydration occurs in adducts $\mathbf{1 7}$ to afford cyclobutenones $\mathbf{1 2 m - t}$.

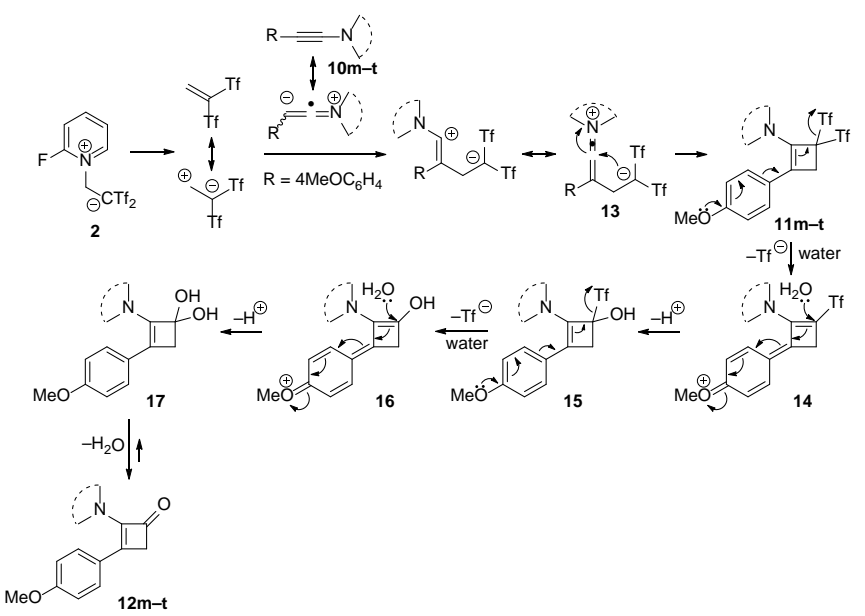

Scheme 9. Rationalization for the formation of cyclobutenones 12.

We decided to examine phosporus-substituted alkynes 18 as precursors of functionalized cyclobutenes (Scheme 10). ${ }^{[11]}$ Screening of precursors 18 revealed that phosphine oxides $18 \mathbf{a}$ and $\mathbf{1 8 b}$ afforded phosphoryl cyclobutenes 19a and 19b in excellent yields under the usual mild conditions. However, despite that apparently thiophosphine oxide $\mathbf{1 8 c}$ is a suitable substrate for the reaction, it produced the $\mathrm{S}=\mathrm{P}(\mathrm{Ph})_{2}$-cyclobutene $19 \mathrm{c}$ in just $15 \%$ yield.

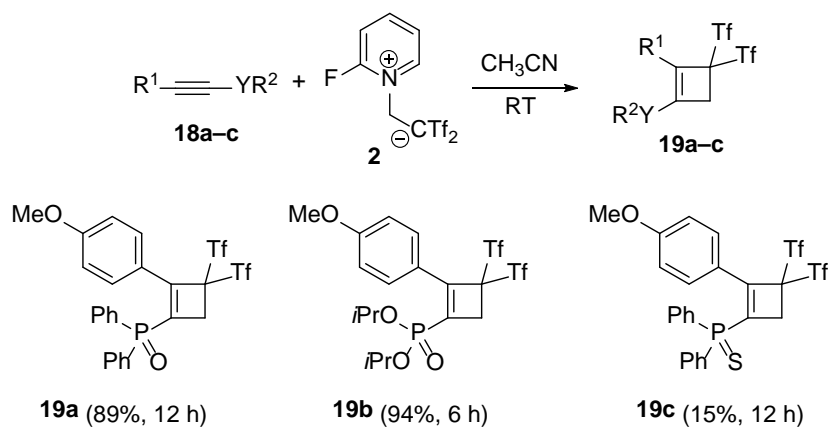

Scheme 10. Controlled preparation of bis(trifluoromethylsulfonyl) phosphoryl cyclobutenes 19ac.

Taking into account the rich chemistry of silicon and tin organoderivatives, we also became interested in the cyclobutenylation of trialkyl(ethynyl)silanes and trialkyl(ethynyl)stannanes by pyridinium salt $\mathbf{2}$ as cyclization reagent. If successfull, this reaction could afford carbon $\left(\mathrm{sp}^{2}\right)$-linked trialkylsilyl- and trialkylstannyl-cyclobutenes. The formation of the expected TMS-cyclobutene 21a was observed by TLC, but it could not be isolated with synthetic purposes. We were pleased to observe that the reaction of the TIPS-alkyne 20b afforded the corresponding TIPScyclobutene 21b in high yield (Scheme 11). An unexpected product was obtained starting from $\mathrm{Bu}_{3} \mathrm{Sn}$-alkyne 22a, which identity was assigned as 23a and resulted from the transformation of the $\mathrm{Bu}_{3} \mathrm{Sn}$ group rather that the alkyne moiety. A similar behaviour was detected in the conversion of organotin derivative 22b into adduct 23b. Interestingly, stirring alkynyl stannane 22c at RT in acetonitrile with zwitterion 2 led to the formation of 
tetra(trifluoromethylsulfonyl)cyclobutene 24c, in 61\% isolated yield in just 10 minutes (Scheme 11). Noticeably, compounds 23a, 23b, and 24c are carbon acids which in solution easily dissociate the acidic hydrogen and give rise to stable carbanions. ${ }^{[12]}$ When the ${ }^{1} \mathrm{H}$ NMR spectra of 23a, 23b, and 24c were performed, the signals for the hydrogen atoms of the $\mathrm{Tf}_{2} \mathrm{CH}$ group could not be detected. Further supporting structural evidence was obtained through the X-ray crystallographic analysis of adduct 24c (Figure 2). ${ }^{[13]}$
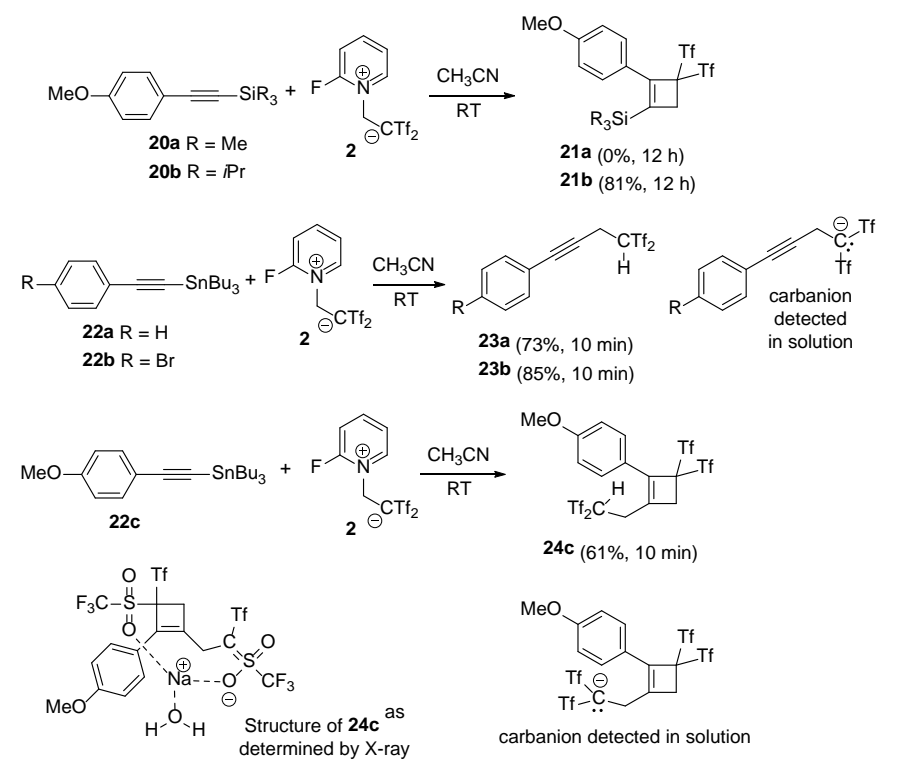

Scheme 11. Controlled preparation of bis(trifluoromethylsulfonyl) silacyclobutene $\mathbf{2 1 b}$ and triflone carbanions 23a,b and 24c.

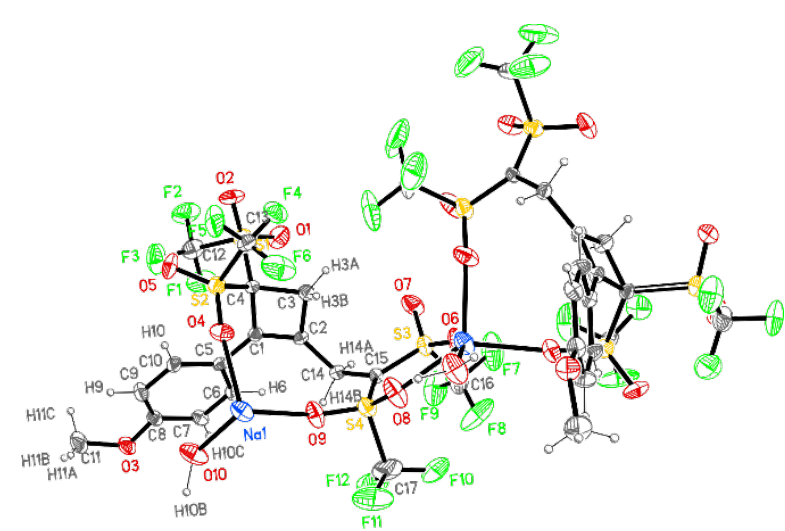

Figure 2. ORTEP drawing of tetra(trifluoromethylsulfonyl)cyclobutene 24c. Thermal ellipsoids shown at 50\% probability.

To evaluate the goal of chemoselectivity, several functionalized heteroatom-containing alkynes 25a-c were reacted under the above standard reaction conditions. Every single reaction reached full conversion to selectively afford cyclobutenes $\mathbf{2 6 a - c}$ in which monocyclization towards the heteroatomsubstituted alkyne was favored. Bis-functionalization of the remaining alkyne or azide functionality was achieved after the addition of a second equivalent of zwitterion 2, suggesting that the exquisite selectivity arises from the increased reactivity imparted by the heteroatom.

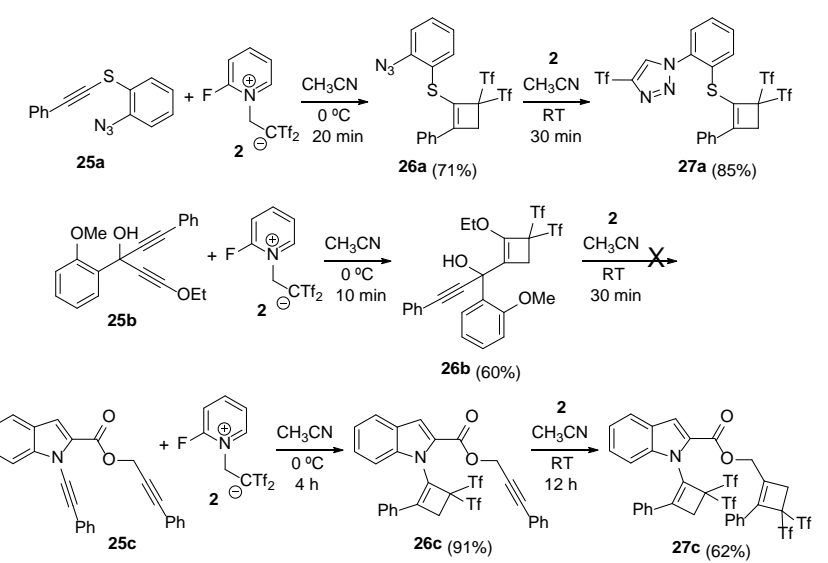

Scheme 12. Chemoselective reaction of heteroatomcontaining alkynes 25 .

\section{Conclusions}

In summary, we have developed a new metal-free synthesis of a vast variety of heteroatom-containing cyclobutene--triflones and cyclobutenones from the reaction of heteroatom-substituted alkynes with a pyridinium salt as a $\mathrm{Tf}_{2} \mathrm{C}=\mathrm{CH}_{2}$ source. This powerful methodology, involving cyclization allows for the selective preparation of oxygen-, nitrogen-, bromine-, chlorine-, iodine-, sulphur-, selenium-, tellurium-, phosphorus-, and silicon-functionalized cyclobutene derivatives.

\section{Experimental Section}

General methods: ${ }^{1} \mathrm{H}$ NMR and ${ }^{13} \mathrm{C}$ NMR spectra were recorded on a Bruker Avance AVIII-700 with cryoprobe, Bruker AMX-500, Bruker Avance-300, or Varian VRX300S. NMR spectra were recorded in $\mathrm{CDCl}_{3}$ solutions, except otherwise stated. Chemical shifts are given in $\mathrm{ppm}$ relative to TMS $\left({ }^{1} \mathrm{H}, 0.0 \mathrm{ppm}\right)$, or $\mathrm{CDCl}_{3}\left({ }^{1} \mathrm{H}, 7.27 \mathrm{ppm} ;{ }^{13} \mathrm{C}\right.$, $76.9 \mathrm{ppm})$, or acetone- $\mathrm{d}_{6}\left({ }^{1} \mathrm{H}, 2.05 \mathrm{ppm} ;{ }^{13} \mathrm{C}, 206.3 \mathrm{ppm}\right)$, or $\mathrm{C}_{6} \mathrm{D}_{6}\left({ }^{1} \mathrm{H}, 7.16 \mathrm{ppm} ;{ }^{13} \mathrm{C}, 128.0 \mathrm{ppm}\right)$, or $\mathrm{CD}_{3} \mathrm{CN}\left({ }^{1} \mathrm{H}\right.$, $\left.1.94 \mathrm{ppm} ;{ }^{13} \mathrm{C}, 118.2 \mathrm{ppm}\right)$, or DMSO-d $6\left({ }^{1} \mathrm{H}, 2.50 \mathrm{ppm} ;{ }^{13} \mathrm{C}\right.$, $39.5 \mathrm{ppm})$. Low and high resolution mass spectra were taken on an AGILENT 6520 Accurate-Mass QTOF LC/MS spectrometer using the electronic impact (EI) or electrospray modes (ES) unless otherwise stated. IR spectra were recorded on a Bruker Tensor 27 spectrometer. X-Ray crystallographic data were collected on a Bruker Smart CCD difractomer using graphite-monochromated Mo-K $\alpha$ radiation $(\lambda=0.71073 \AA$ ) operating at $50 \mathrm{Kv}$ and $35 \mathrm{~mA}$ with an exposure of $30.18 \mathrm{~s}$ in $\omega$. All commercially available compounds were used without further purification.

General procedure for the reaction of heteroatomsubstituted alkynes and pyridinium salt 2. 2-(2Fluoropyridin-1-ium-1-yl)-1,1-

bis[(trifluoromethyl)sulfonyl]ethan-1-ide 2 (0.2 mmol) was added at room temperature (or $0{ }^{\circ} \mathrm{C}$ ) to a solution of the 
appropriate heteroatom-substituted alkyne 1a-f, 4a-u, 8a-i, 10a-l, 18a-c, 20a, 20b, 22a-c, 25a-c, 26a-c (0.2 mmol) in acetonitrile $(4 \mathrm{~mL})$. The reaction was stirred at room temperature until disappearance of the starting material (TLC), and then the mixture was concentrated under reduced pressure. Chromatography of the residue eluting with hexanes/ethyl acetate mixtures gave analytically pure compounds. Spectroscopic and analytical data for adducts 3a-f, 5a-p, 6q-u, 9c-i, 11a-l, 19a-c, 21b, 23a, 23b, 24c, 26a-c, 271, 27c follow. ${ }^{[14]}$

Bis(trifluoromethylsulfonyl)iodocyclobutene 3c. From $43 \mathrm{mg}$ (0.16 mmol) of alkyne 1c, and after flash chromatography of the residue using hexanes/ethyl acetate (97:3) as eluent gave compound 3c (153 mg, 97\%) as a pale yellow solid; mp 83-85 ${ }^{\circ} \mathrm{C}$; ${ }^{1} \mathrm{H}$ NMR (300 MHz, $\mathrm{CDCl}_{3}, 25$ $\left.{ }^{\circ} \mathrm{C}\right): \delta=7.91\left(\mathrm{~m}, 2 \mathrm{H}, 2 \mathrm{CH}^{\mathrm{Ar}}\right), 6.98\left(\mathrm{~m}, 2 \mathrm{H}, 2 \mathrm{CH}^{\mathrm{Ar}}\right), 3.86(\mathrm{~s}$, $\left.3 \mathrm{H}, \mathrm{OCH}_{3}\right), 3.67$ (s, $\left.2 \mathrm{H}, \mathrm{CH}_{2}\right)$; ${ }^{13} \mathrm{C} \mathrm{NMR}\left(75 \mathrm{MHz} \mathrm{CDCl}_{3}\right.$, $\left.25{ }^{\circ} \mathrm{C}\right): \delta=161.5\left(C^{\mathrm{Ar}-\mathrm{q}}-\mathrm{OCH}_{3}\right), 142.4(\mathrm{C}=\mathrm{C}-\mathrm{I}), 128.7$ $\left(2 \mathrm{CH}^{\mathrm{Ar}}\right), 121.2\left(\mathrm{C}^{\mathrm{Ar}-\mathrm{q}}\right), 119.7\left(\mathrm{q}, J_{C F}=331.5 \mathrm{~Hz}, 2 \mathrm{CF}_{3}\right)$, $114.1\left(2 \mathrm{CH}^{\mathrm{Ar}}\right), 93.7(\mathrm{C}=\mathrm{C}-\mathrm{I}), 90.0\left(\mathrm{CTf}_{2}\right), 55.4\left(\mathrm{CH}_{3} \mathrm{O}\right)$, $42.5\left(\mathrm{CH}_{2}\right) ;{ }^{19} \mathrm{~F} \mathrm{NMR}\left(282 \mathrm{MHz}, \mathrm{CDCl}_{3}, 25^{\circ} \mathrm{C}\right): \delta=-70.61$ (s, 6F, 2CF 3$)$; IR $\left(\mathrm{CHCl}_{3}\right): v=1608(\mathrm{C}=\mathrm{C}), 1384,1103$ $(\mathrm{O}=\mathrm{S}=\mathrm{O}), 1207$ (C-F) cm $\mathrm{cm}^{-1}$; HRMS (ES): calcd for $\mathrm{C}_{13} \mathrm{H}_{13} \mathrm{IF}_{6} \mathrm{NO}_{5} \mathrm{~S}_{2}\left[M+\mathrm{NH}_{4}\right]^{+}$: 567.9179; found: 567.9177.

Bis(trifluoromethylsulfonyl)phenoxycyclobutene $5 d$. From $20 \mathrm{mg}(0.11 \mathrm{mmol})$ of alkyne $\mathbf{4 d}$, and after flash chromatography of the residue using hexanes/toluene $(9: 1 \rightarrow 8: 2)$ as eluent gave compound $5 \mathbf{d}$ (36 $\mathrm{mg}, 68 \%)$ as a colorless oil; ${ }^{1} \mathrm{H}$ NMR (300 MHz, $\left.\mathrm{CDCl}_{3}, 25{ }^{\circ} \mathrm{C}\right): \delta=7.40$ $\left(\mathrm{m}, 2 \mathrm{H}, 2 \mathrm{CH}^{\mathrm{Ar}}\right), 7.22\left(\mathrm{~m}, 3 \mathrm{H}, 3 \mathrm{CH}^{\mathrm{Ar}}\right), 2.98\left(\mathrm{~s}, 2 \mathrm{H}, \mathrm{CH}_{2}-\right.$ cyclobutenyl), 1.77 (t, 3H, $J=7.2 \mathrm{~Hz}, \mathrm{CH}_{2}$ ), 1.24 (m, 4H, $\left.2 \mathrm{CH}_{2}\right), 0.79\left(\mathrm{t}, 3 \mathrm{H}, J=7.1 \mathrm{~Hz}, \mathrm{CH}_{3}\right) ;{ }^{13} \mathrm{C}$ NMR $(75 \mathrm{MHz}$, $\left.\mathrm{CDCl}_{3}, 2{ }^{\circ} \mathrm{C}\right): \delta=154.1$ ( $\left.\mathrm{C}^{\mathrm{Ar}-\mathrm{q}}-\mathrm{O}\right), 133.7(\mathrm{C}=\mathrm{C}-\mathrm{O}), 132.5$ $(C=\mathrm{C}-\mathrm{O}), 129.9\left(2 \mathrm{CH}^{\mathrm{Ar}}\right), 125.6\left(\mathrm{CH}^{\mathrm{Ar}}\right), 119.7\left(\mathrm{q}, J_{C F}=\right.$ $\left.330.5 \mathrm{~Hz}, 2 \mathrm{CF}_{3}\right), 119.0\left(2 \mathrm{CH}^{\mathrm{Ar}}\right), 86.8\left(\mathrm{CTf}_{2}\right), 30.0\left(\mathrm{CH}_{2}-\right.$ cyclobutenyl), $27.8\left(\mathrm{CH}_{2}\right), 27.0\left(\mathrm{CH}_{2}\right), 22.1\left(\mathrm{CH}_{2}\right), 13.5$ $\left(\mathrm{CH}_{3}\right) ;{ }^{19} \mathrm{~F}$ NMR (282 MHz, $\left.\mathrm{CDCl}_{3}, 25{ }^{\circ} \mathrm{C}\right): \delta=-70.91(\mathrm{~s}$, $\left.6 \mathrm{~F}, 2 \mathrm{CF}_{3}\right)$; IR $\left(\mathrm{CHCl}_{3}\right): v=1695(\mathrm{C}=\mathrm{C}), 1383,1106$ $(\mathrm{O}=\mathrm{S}=\mathrm{O}), 1204$ (C-F) $\mathrm{cm}^{-1}$; HRMS (ES): calcd for $\mathrm{C}_{16} \mathrm{H}_{20} \mathrm{~F}_{6} \mathrm{NO}_{5} \mathrm{~S}_{2}\left[M+\mathrm{NH}_{4}\right]^{+}$: 484.0682; found: 484.0671.

Bis(trifluoromethylsulfonyl)thiocyclobutene 5f. From 30 $\mathrm{mg}(0.103 \mathrm{mmol})$ of alkyne 4f, and after flash chromatography of the residue using hexanes/ethyl acetate (95:5) as eluent gave compound 5 f (56 mg, 93\%) as a colorless oil; ${ }^{1} \mathrm{H}$ NMR (300 MHz, $\left.\mathrm{C}_{6} \mathrm{D}_{6}, 25^{\circ} \mathrm{C}\right): \delta=7.25$ $\left(\mathrm{m}, 2 \mathrm{H}, 2 \mathrm{CH}^{\mathrm{Ar}}\right), 6.96\left(\mathrm{~m}, 2 \mathrm{H}, 2 \mathrm{CH}^{\mathrm{Ar}}\right), 6.79\left(\mathrm{~m}, 5 \mathrm{H}, 5 \mathrm{CH}^{\mathrm{Ar}}\right)$, 3.17 (s, $\left.2 \mathrm{H}, \mathrm{CH}_{2}\right) ;{ }^{13} \mathrm{C}$ NMR $\left(75 \mathrm{MHz}, \mathrm{C}_{6} \mathrm{D}_{6}, 25^{\circ} \mathrm{C}\right): \delta=$ $159.0(\mathrm{C}=\mathrm{C}-\mathrm{S}), 131.9\left(2 \mathrm{CH}^{\mathrm{Ar}}\right), 130.3\left(C^{\mathrm{Ar}-\mathrm{q}}\right), 129.7\left(2 \mathrm{CH}^{\mathrm{Ar}}\right)$, $129.6\left(2 \mathrm{CH}^{\mathrm{Ar}}\right), 129.5\left(2 \mathrm{CH}^{\mathrm{Ar}}\right), 128.6\left(\mathrm{C}^{\mathrm{Ar}-\mathrm{q}}\right), 128.0\left(\mathrm{CH}^{\mathrm{Ar}}\right)$, $126.9\left(\mathrm{C}^{\mathrm{Ar}-\mathrm{q}}\right), 120.3\left(\mathrm{q}, J_{C F}=331.1 \mathrm{~Hz}, 2 \mathrm{CF}_{3}\right), 121.0(C=\mathrm{C}-$ $\mathrm{S}), 89.2\left(\mathrm{CTf}_{2}\right), 34.8\left(\mathrm{CH}_{2}\right) ;{ }^{19} \mathrm{~F}$ NMR (282 MHz, $\mathrm{C}_{6} \mathrm{D}_{6}, 25$ $\left.{ }^{\circ} \mathrm{C}\right): \delta=-70.29\left(\mathrm{~s}, 6 \mathrm{~F}, 2 \mathrm{CF}_{3}\right)$; IR $\left(\mathrm{CH}_{2} \mathrm{Cl}_{2}\right): v=1599(\mathrm{C}=\mathrm{C})$, 1383, $1106(\mathrm{O}=\mathrm{S}=\mathrm{O}), 1207$ (C-F) cm ${ }^{-1}$; HRMS (ES): calcd for $\mathrm{C}_{18} \mathrm{H}_{15} \mathrm{BrF}_{6} \mathrm{NO}_{4} \mathrm{~S}_{3}\left[M+\mathrm{NH}_{4}\right]^{+}$: 597.9245; found: 597.9231.

Bis(trifluoromethylsulfonyl)selenocyclobutene $5 \mathrm{~m}$. From $30 \mathrm{mg}(0.12 \mathrm{mmol})$ of alkyne $\mathbf{4 m}$, and after flash chromatography of the residue using hexanes $\rightarrow$ hexanes/ethyl acetate (95:5) as eluent gave compound $5 \mathrm{~m}$ (64 mg, quantitative yield) as a colorless oil; ${ }^{1} \mathrm{H}$ NMR (300 MHz, $\left.\mathrm{CDCl}_{3}, 25{ }^{\circ} \mathrm{C}\right): \delta=7.82(\mathrm{~m}, 2 \mathrm{H}$, $\left.2 \mathrm{CH}^{\mathrm{Ar}}\right), 7.51\left(\mathrm{~m}, 3 \mathrm{H}, 3 \mathrm{CH}^{\mathrm{Ar}}\right), 3.76\left(\mathrm{~s}, 2 \mathrm{H}, \mathrm{CH}_{2}-\right.$ cyclobutenyl), 3.04 (t, 2H, J = 7.5 Hz, $\left.\mathrm{CH}_{2}\right), 1.73(\mathrm{~m}, 2 \mathrm{H}$, $\left.\mathrm{CH}_{2}\right), 1.33\left(\mathrm{~m}, 4 \mathrm{H}, 2 \mathrm{CH}_{2}\right), 0.86\left(\mathrm{t}, 3 \mathrm{H}, J=7.1 \mathrm{~Hz}, \mathrm{CH}_{3}\right)$; ${ }^{13} \mathrm{C}$ NMR (75 MHz, $\left.\mathrm{CDCl}_{3}, 25{ }^{\circ} \mathrm{C}\right): \delta=161.6$ ( $\left.\mathrm{C}=\mathrm{C}-\mathrm{Se}\right)$, $131.9\left(\mathrm{CH}^{\mathrm{Ar}}\right), 130.7\left(\mathrm{C}^{\mathrm{Ar}-\mathrm{q}}\right), 128.9\left(2 \mathrm{CH}^{\mathrm{Ar}}\right), 127.4\left(2 \mathrm{CH}^{\mathrm{Ar}}\right)$, $119.8\left(\mathrm{q}, J_{C F}=331.3 \mathrm{~Hz}, 2 \mathrm{CF}_{3}\right), 115.0(\mathrm{C}=C-\mathrm{Se}), 87.8$ $\left(\mathrm{CTf}_{2}\right), 36.4\left(\mathrm{CH}_{2}\right.$-cyclobutenyl), $31.7\left(\mathrm{CH}_{2}\right), 30.0\left(\mathrm{CH}_{2}\right)$, $29.5\left(\mathrm{CH}_{2}\right), 22.0\left(\mathrm{CH}_{2}\right), 13.8\left(\mathrm{CH}_{3}\right) ;{ }^{19} \mathrm{~F}$ NMR $(282 \mathrm{MHz}$, $\left.\mathrm{CDCl}_{3}, 25{ }^{\circ} \mathrm{C}\right): \delta=-70.23$ (s, 6F, 2CF $)$; ${ }^{77} \mathrm{Se} \mathrm{NMR}(95$ $\left.\mathrm{MHz}, \mathrm{CDCl}_{3}, 25^{\circ} \mathrm{C}\right): \delta=245.0$ (s, 1Se, Se); IR $\left(\mathrm{CHCl}_{3}\right): v$ $=1381,1106(\mathrm{O}=\mathrm{S}=\mathrm{O}), 1203(\mathrm{C}-\mathrm{F}) \mathrm{cm}^{-1}$; HRMS (ES): calcd for $\mathrm{C}_{17} \mathrm{H}_{22} \mathrm{~F}_{6} \mathrm{NO}_{4} \mathrm{~S}_{2}$ Se $\left[M+\mathrm{NH}_{4}\right]^{+}$: 562.0054; found: 562.0037.

Bis(trifluoromethylsulfonyl)telurocyclobutene 5p. From $50 \mathrm{mg}(0.178 \mathrm{mmol})$ of alkyne $\mathbf{4 p}$, and after flash chromatography of the residue using hexanes/ethyl acetate (95:5) as eluent gave compound 5p (102 mg, quantitative yield) as a pale yellow oil; ${ }^{1} \mathrm{H}$ NMR (300 $\mathrm{MHz} \mathrm{CDCl}_{3}, 25$ $\left.{ }^{\circ} \mathrm{C}\right): \delta=3.62$ (s, $2 \mathrm{H}, \mathrm{CH}_{2}$-cyclobutenyl), 2.90 (t, $2 \mathrm{H}, J=7.6$ $\left.\mathrm{Hz}, \mathrm{CH}_{2}\right), 2.43$ (t, 2H, J = 7.4 Hz, $\left.\mathrm{CH}_{2}\right), 1.82\left(\mathrm{~m}, 2 \mathrm{H}, \mathrm{CH}_{2}\right)$, $1.41\left(\mathrm{~m}, 8 \mathrm{H}, 4 \mathrm{CH}_{2}\right), 0.91\left(\mathrm{~m}, 6 \mathrm{H}, 2 \mathrm{CH}_{3}\right) ;{ }^{13} \mathrm{C}$ NMR $(75$ $\left.\mathrm{MHz}, \mathrm{CDCl}_{3}, 25^{\circ} \mathrm{C}\right): \delta=178.6$ (C=C-Te), 119.9 (q, $J_{C F}=$ $\left.331.1 \mathrm{~Hz}, 2 \mathrm{CF}_{3}\right), 98.3(\mathrm{C}=\mathrm{C}-\mathrm{Te}), 86.5\left(\mathrm{CTf}_{2}\right), 39.8\left(\mathrm{CH}_{2}-\right.$ cyclobutenyl), $33.9\left(\mathrm{CH}_{2}\right), 31.8\left(\mathrm{CH}_{2}\right), 31.3\left(\mathrm{CH}_{2}\right), 28.1$ $\left(\mathrm{CH}_{2}\right), 22.3\left(\mathrm{CH}_{2}\right), 22.0\left(\mathrm{CH}_{2}\right), 13.9\left(\mathrm{CH}_{3}\right), 13.7\left(\mathrm{CH}_{3}\right)$, $10.5\left(\mathrm{CH}_{2}\right) ;{ }^{19} \mathrm{~F} \mathrm{NMR}\left(282 \mathrm{MHz}, \mathrm{CDCl}_{3}, 25^{\circ} \mathrm{C}\right): \delta=-70.27$ (s, 6F, 2- $\left.\mathrm{CF}_{3}\right)$; IR $\left(\mathrm{CHCl}_{3}\right): v=1605(\mathrm{C}=\mathrm{C}), 1380,1107$ $(\mathrm{O}=\mathrm{S}=\mathrm{O}), 1203$ (C-F) $\mathrm{cm}^{-1}$; HRMS (ES): calcd for $\mathrm{C}_{15} \mathrm{H}_{26} \mathrm{~F}_{6} \mathrm{O}_{4} \mathrm{~S}_{2} \mathrm{Te}\left[M+\mathrm{NH}_{4}\right]^{+}:$592.0260; found: 592.0254

Thiocyclobutenone $6 s$. From $30 \mathrm{mg}$ ( $0.12 \mathrm{mmol})$ of alkyne 4s, and after flash chromatography of the residue using hexanes/ethyl acetate (97:3) as eluent gave compound $\mathbf{6 s}$ (15 mg, 42\%) as a pale yellow oil; ${ }^{1} \mathrm{H}$ NMR (300 MHz, $\left.\mathrm{CDCl}_{3}, 25^{\circ} \mathrm{C}\right): \delta=7.37\left(\mathrm{~m}, 10 \mathrm{H}, 10 \mathrm{CH}^{\mathrm{Ar}}\right), 3.31(\mathrm{~s}, 2 \mathrm{H}$, $\left.\mathrm{CH}_{2}\right) ;{ }^{13} \mathrm{C} \mathrm{NMR}\left(75 \mathrm{MHz}, \mathrm{CDCl}_{3}, 25^{\circ} \mathrm{C}\right): \delta=182.0(\mathrm{C}=\mathrm{O})$, $172.9(C=\mathrm{C}), 134.1\left(2 \mathrm{CH}^{\mathrm{Ar}}\right), 131.6\left(C^{\mathrm{Ar}-\mathrm{q}}\right), 130.4\left(2 \mathrm{CH}^{\mathrm{Ar}}\right)$, $130.1\left(\mathrm{CH}^{\mathrm{Ar}}\right), 129.6\left(\mathrm{C}^{\mathrm{Ar}-\mathrm{q}}\right), 129.4\left(2 \mathrm{CH}^{\mathrm{Ar}}\right), 129.0\left(2 \mathrm{CH}^{\mathrm{Ar}}\right)$, $128.4(\mathrm{C}=\mathrm{C}), 127.2\left(\mathrm{CH}^{\mathrm{Ar}}\right), 52.3\left(\mathrm{CH}_{2}\right)$; IR $\left(\mathrm{CHCl}_{3}\right): v=$ $1742(\mathrm{C}=\mathrm{O}) \mathrm{cm}^{-1}$; HRMS (ES): calcd for $\mathrm{C}_{16} \mathrm{H}_{13} \mathrm{OS}_{2}[M+$ $\mathrm{H}]^{+}$: 285.0402; found: 285.0410.

Bis(trifluoromethylsulfonyl)sulfinylcyclobutene 9 9f. From $15 \mathrm{mg}(0.064 \mathrm{mmol})$ of alkyne 8f, and after flash chromatography of the residue using hexanes/ethyl acetate $(9: 1 \rightarrow 8: 2)$ as eluent gave compound $\mathbf{9 f}(18 \mathrm{mg}, 53 \%)$ as a pale yellow oil; ${ }^{1} \mathrm{H}$ NMR (700 MHz, $\left.\mathrm{C}_{6} \mathrm{D}_{6}, 25^{\circ} \mathrm{C}\right): \delta=7.92$ (d, $\left.1 \mathrm{H}, J=3.1 \mathrm{~Hz}, \mathrm{CH}^{\mathrm{Ar}}\right), 7.53\left(\mathrm{~m}, 2 \mathrm{H}, 2 \mathrm{CH}^{\mathrm{Ar}}\right), 6.95(\mathrm{~m}, 3 \mathrm{H}$, $3 \mathrm{CH}^{\mathrm{Ar}}$ ), $6.67\left(\mathrm{~d}, 1 \mathrm{H}, J=5.0 \mathrm{~Hz}, \mathrm{CH}^{\mathrm{Ar}}\right), 6.38(\mathrm{t}, 1 \mathrm{H}, J=4.4$ $\mathrm{Hz}, \mathrm{CH}^{\mathrm{Ar}}$ ), 3.53 (d, $1 \mathrm{H}, J=15.7 \mathrm{~Hz}, \mathrm{CH} H$-cyclobutenyl), $2.93\left(\mathrm{~d}, 1 \mathrm{H}, J=15.7 \mathrm{~Hz}, \mathrm{CHH}\right.$-cyclobutenyl); ${ }^{13} \mathrm{C} \mathrm{NMR}$ (175 MHz, $\left.\mathrm{C}_{6} \mathrm{D}_{6}, 25^{\circ} \mathrm{C}\right): \delta=146.0(\mathrm{C}=\mathrm{C}-\mathrm{S}), 141.0\left(\mathrm{C}^{\mathrm{Ar}-\mathrm{q}}\right)$, $134.1\left(\mathrm{CH}^{\mathrm{Ar}}\right), 132.3(\mathrm{C}=\mathrm{C}-\mathrm{S}), 132.2\left(\mathrm{CH}^{\mathrm{Ar}}\right), 131.9\left(\mathrm{CH}^{\mathrm{Ar}}\right)$, $129.7\left(2 \mathrm{CH}^{\mathrm{Ar}}\right), 128.7\left(\mathrm{CH}^{\mathrm{Ar}}\right), 127.6\left(\mathrm{C}^{\mathrm{Ar}-\mathrm{q}}\right), 124.1\left(2 \mathrm{CH}^{\mathrm{Ar}}\right)$, $120.2\left(\mathrm{q}, J_{C F}=331.8 \mathrm{~Hz}, \mathrm{CF}_{3}\right), 119.9\left(\mathrm{q}, J_{C F}=331.6 \mathrm{~Hz}\right.$, $\left.\mathrm{CF}_{3}\right)$, $84.4\left(\mathrm{CTf}_{2}\right), 33.7\left(\mathrm{CH}_{2}\right) ;{ }^{19} \mathrm{~F}$ NMR (282 MHz, $\mathrm{C}_{6} \mathrm{D}_{6}$, $\left.25^{\circ} \mathrm{C}\right): \delta=-70.74\left(\mathrm{~s}, 3 \mathrm{~F}, \mathrm{CF}_{3}\right),-71.63\left(\mathrm{~s}, 3 \mathrm{~F}, \mathrm{CF}_{3}\right)$; IR $\left(\mathrm{CH}_{2} \mathrm{Cl}_{2}\right): v=1386,1105(\mathrm{O}=\mathrm{S}=\mathrm{O}), 1212(\mathrm{C}-\mathrm{F}) \mathrm{cm}^{-1}$; 
HRMS (ES): calcd for $\mathrm{C}_{16} \mathrm{H}_{11} \mathrm{~F}_{6} \mathrm{O}_{5} \mathrm{~S}_{4}[M+\mathrm{H}]^{+}$: 524.9388; found: 524.9400 .

\section{Bis(trifluoromethylsulfonyl)sulfonylcyclobutene}

9i. From $44 \mathrm{mg}(0.16 \mathrm{mmol})$ of alkyne $\mathbf{8 i}$, and after flash chromatography of the residue using hexanes/ethyl acetate (97:3) as eluent gave compound $\mathbf{9 i}$ (90 $\mathrm{mg}$, quantitative yield) as a colorless solid; mp 103-105 ${ }^{\circ} \mathrm{C}$; ${ }^{1} \mathrm{H}$ NMR (300 $\left.\mathrm{MHz} \mathrm{CDCl}_{3}, 25^{\circ} \mathrm{C}\right): \delta=8.07\left(\mathrm{~m}, 2 \mathrm{H}, 2 \mathrm{CH}^{\mathrm{Ar}}\right), 7.93(\mathrm{~m}, 2 \mathrm{H}$, $\left.2 \mathrm{CH}^{\mathrm{Ar}}\right), 7.71\left(\mathrm{~m}, 1 \mathrm{H}, \mathrm{CH}^{\mathrm{Ar}}\right), 7.59\left(\mathrm{~m}, 2 \mathrm{H}, 2 \mathrm{CH}^{\mathrm{Ar}}\right), 6.97(\mathrm{~m}$, $\left.2 \mathrm{H}, 2 \mathrm{CH}^{\mathrm{Ar}}\right), 3.88\left(\mathrm{~s}, 3 \mathrm{H}, \mathrm{OCH}_{3}\right), 3.50\left(\mathrm{~s}, 2 \mathrm{H}, \mathrm{CH}_{2}\right) ;{ }^{13} \mathrm{C}$ NMR (75 MHz, $\left.\mathrm{CDCl}_{3}, 25{ }^{\circ} \mathrm{C}\right): \delta=163.0\left(C^{\mathrm{Ar}-\mathrm{q}}-\mathrm{OCH}_{3}\right)$, $141.9\left(C=\mathrm{C}-\mathrm{SO}_{2} \mathrm{Ph}\right), 139.1\left(\mathrm{C}=\mathrm{C}-\mathrm{SO}_{2} \mathrm{Ph}\right), 137.5\left(\mathrm{C}^{\mathrm{Ar}-\mathrm{q}}\right)$, $135.0\left(\mathrm{CH}^{\mathrm{Ar}}\right), 132.5\left(2 \mathrm{CH}^{\mathrm{Ar}}\right), 129.7\left(2 \mathrm{CH}^{\mathrm{Ar}}\right), 127.9\left(2 \mathrm{CH}^{\mathrm{Ar}}\right)$, $119.6\left(\mathrm{q}, J_{C F}=331.5 \mathrm{~Hz}, 2 \mathrm{CF}_{3}\right), 119.5\left(\mathrm{C}^{\mathrm{Ar}-\mathrm{q}}\right), 114.3$ $\left(2 \mathrm{CH}^{\mathrm{Ar}}\right), 84.2\left(\mathrm{CTf}_{2}\right), 55.5\left(\mathrm{OCH}_{3}\right), 35.9\left(\mathrm{CH}_{2}\right) ;{ }^{19} \mathrm{~F}$ NMR (282 MHz, $\left.\mathrm{CDCl}_{3}, 25{ }^{\circ} \mathrm{C}\right): \delta=-70.51$ (s, 6F, 2CF $)$; IR $\left(\mathrm{CHCl}_{3}\right): v=1599(\mathrm{C}=\mathrm{C}), 1386,1101(\mathrm{O}=\mathrm{S}=\mathrm{O}), 1212(\mathrm{C}-$ F) $\mathrm{cm}^{-1}$; HRMS (ES): calcd for $\mathrm{C}_{19} \mathrm{H}_{18} \mathrm{~F}_{6} \mathrm{NO}_{7} \mathrm{~S}_{3}[M+$ $\left.\mathrm{NH}_{4}\right]^{+}$: 582.0144; found: 582.0155 .

Bis(trifluoromethylsulfonyl)aminocyclobutene 11a. From $20 \mathrm{mg}(0.07 \mathrm{mmol})$ of alkyne 10a, and after flash chromatography of the residue using hexanes/ethyl acetate (95:5) as eluent gave compound 11a (38 mg, 94\%) as a colorless solid; mp 117-119 ${ }^{\circ} \mathrm{C}$; ${ }^{1} \mathrm{H}$ NMR (300 MHz, $\mathrm{C}_{6} \mathrm{D}_{6}$, $\left.25^{\circ} \mathrm{C}\right): \delta=8.65$ (d, $\left.1 \mathrm{H}, J=8.0 \mathrm{~Hz}, \mathrm{CH}^{\mathrm{Ar}}\right), 8.35$ (s, $1 \mathrm{H}$, $\left.\mathrm{NCH}^{\mathrm{Ar}}\right), 7.40\left(\mathrm{~d}, 1 \mathrm{H}, J=8.3 \mathrm{~Hz}, \mathrm{CH}^{\mathrm{Ar}}\right), 7.15\left(\mathrm{~m}, 1 \mathrm{H}, \mathrm{CH}^{\mathrm{Ar}}\right)$, $6.98\left(\mathrm{~m}, 1 \mathrm{H}, \mathrm{CH}^{\mathrm{Ar}}\right), 6.84\left(\mathrm{~m}, 1 \mathrm{H}, \mathrm{CH}^{\mathrm{Ar}}\right), 6.65(\mathrm{~m}, 4 \mathrm{H}$, $4 \mathrm{CH}^{\mathrm{Ar}}$ ), 3.48 (s, 3H, $\left.\mathrm{CH}_{3}\right), 3.11$ (s, $\left.2 \mathrm{H}, \mathrm{CH}_{2}\right) ;{ }^{13} \mathrm{C}$ NMR (75 $\left.\mathrm{MHz}, \mathrm{C}_{6} \mathrm{D}_{6}, 25^{\circ} \mathrm{C}\right): \delta=164.0(\mathrm{C}=\mathrm{O}), 152.9(\mathrm{C}=\mathrm{C}-\mathrm{N})$, 136.4 $(C=\mathrm{C}-\mathrm{N}), 132.5\left(\mathrm{CH}^{\mathrm{Ar}}\right), 131.7\left(\mathrm{CH}^{\mathrm{Ar}}\right), 129.1\left(2 \mathrm{CH}^{\mathrm{Ar}}\right)$, $128.4\left(2 \mathrm{CH}^{\mathrm{Ar}}\right), 128.2\left(\mathrm{C}^{\mathrm{Ar}-\mathrm{q}}\right), 126.9\left(\mathrm{C}^{\mathrm{Ar}-\mathrm{q}}\right), 125.0\left(\mathrm{CH}^{\mathrm{Ar}}\right)$, $124.0\left(\mathrm{CH}^{\mathrm{Ar}}\right), 123.0\left(\mathrm{CH}^{\mathrm{Ar}}\right), 120.1\left(\mathrm{q}, J_{C F}=330.8 \mathrm{~Hz}, 2 \mathrm{CF}_{3}\right)$, $118.9\left(\mathrm{C}^{\mathrm{Ar}-\mathrm{q}}\right), 112.8\left(\mathrm{C}^{\mathrm{Ar}-\mathrm{qo}}\right), 112.0\left(\mathrm{CH}^{\mathrm{Ar}}\right), 90.0\left(\mathrm{CTf}_{2}\right), 51.0$ $\left(\mathrm{CH}_{3}\right), 31.1\left(\mathrm{CH}_{2}\right) ;{ }^{19} \mathrm{~F}$ NMR $\left(282 \mathrm{MHz}, \mathrm{C}_{6} \mathrm{D}_{6}, 25{ }^{\circ} \mathrm{C}\right): \delta=$ -70.44 (s, 6F, 2CF $)$; IR $\left(\mathrm{CH}_{2} \mathrm{Cl}_{2}\right): v=1711(\mathrm{C}=\mathrm{O}), 1375$, $1102(\mathrm{O}=\mathrm{S}=\mathrm{O}), 1196(\mathrm{C}-\mathrm{F}) \mathrm{cm}^{-1}$; HRMS (ES): calcd for $\mathrm{C}_{22} \mathrm{H}_{19} \mathrm{~F}_{6} \mathrm{~N}_{2} \mathrm{O}_{6} \mathrm{~S}_{2}\left[M+\mathrm{NH}_{4}\right]^{+}$: 585.0583; found: 585.0559.

\section{Bis(trifluoromethylsulfonyl)phosphinylcyclobutene $19 \mathrm{~b}$.} From $53 \mathrm{mg}(0.18 \mathrm{mmol})$ of alkyne $\mathbf{1 8 b}$, and after flash chromatography of the residue using hexanes/ethyl acetate $(95: 5 \rightarrow 9: 1)$ as eluent gave compound $19 b$ (99 $\mathrm{mg}, 94 \%)$ as a colorless oil; ${ }^{1} \mathrm{H}$ NMR $\left(300 \mathrm{MHz}, \mathrm{CDCl}_{3}, 25^{\circ} \mathrm{C}\right): \delta=8.06$ $\left(\mathrm{m}, 2 \mathrm{H}, 2 \mathrm{CH}^{\mathrm{Ar}}\right), 6.95\left(\mathrm{~m}, 2 \mathrm{H}, 2 \mathrm{CH}^{\mathrm{Ar}}\right), 4.76(\mathrm{~m}, 2 \mathrm{H}, 2 \mathrm{CH})$, 3.85 (s, $3 \mathrm{H}, \mathrm{OCH}_{3}$ ), 3.50 (s, $2 \mathrm{H}, \mathrm{CH}_{2}$ ), 1.37 (d, $6 \mathrm{H}, J=6.2$ $\left.\mathrm{Hz}, 2 \mathrm{CH}_{3}\right), 1.27$ (d, $\left.6 \mathrm{H}, J=6.2 \mathrm{~Hz}, 2 \mathrm{CH}_{3}\right) ;{ }^{13} \mathrm{C}$ NMR $(75$ $\left.\mathrm{MHz}, \mathrm{CDCl}_{3}, 25^{\circ} \mathrm{C}\right): \delta=162.1\left(C^{\mathrm{Ar}-\mathrm{q}}-\mathrm{OCH}_{3}\right), 145.6\left(\mathrm{~d}, J_{C P}\right.$ $=9.9 \mathrm{~Hz}, C=\mathrm{C}-\mathrm{P}), 136.6\left(\mathrm{~d}, J_{C P}=188.7 \mathrm{~Hz}, \mathrm{C}=C-\mathrm{P}\right), 131.1$ $\left(2 \mathrm{CH}^{\mathrm{Ar}}\right), 121.5\left(\mathrm{C}^{\mathrm{Ar}-\mathrm{q}}\right), 119.8\left(\mathrm{q}, J_{C F}=331.6 \mathrm{~Hz}, 2 \mathrm{CF}_{3}\right)$, $114.0\left(2 \mathrm{CH}^{\mathrm{Ar}}\right), 86.3\left(\mathrm{~d}, J_{C P}=35.8 \mathrm{~Hz}, \mathrm{CTf}_{2}\right), 72.5\left(\mathrm{~d}, J_{C P}=\right.$ $5.6 \mathrm{~Hz}, 2 \mathrm{CH}), 55.3\left(\mathrm{OCH}_{3}\right), 36.0\left(\mathrm{~d}, J_{C P}=7.9 \mathrm{~Hz}, \mathrm{CH}_{2}\right)$, 24.0 (d, $\left.J_{C P}=4.1 \mathrm{~Hz}, 2 \mathrm{CH}_{3}\right), 23.7$ (d, $\left.J_{C P}=5.0 \mathrm{~Hz}, 2 \mathrm{CH}_{3}\right)$; ${ }^{19} \mathrm{~F}$ NMR (282 MHz, $\mathrm{CDCl}_{3}, 25{ }^{\circ} \mathrm{C}$ ): $\delta=-70.57$ (s, 6F, $\left.2 \mathrm{CF}_{3}\right) ;{ }^{31} \mathrm{P} \mathrm{NMR}\left(121 \mathrm{MHz}, \mathrm{CDCl}_{3}, 25{ }^{\circ} \mathrm{C}\right): \delta=4.48[\mathrm{~s}, \mathrm{P}$, $\left.\mathrm{P}=\mathrm{O}\left(\mathrm{O}^{\mathrm{i} P r}\right)_{2}\right]$; IR $\left(\mathrm{CHCl}_{3}\right): \mathrm{v}=1605(\mathrm{C}=\mathrm{C}), 1387,1103$ $(\mathrm{O}=\mathrm{S}=\mathrm{O}), 1206(\mathrm{C}-\mathrm{F}), 988(\mathrm{P}=\mathrm{O}) \mathrm{cm}^{-1}$; HRMS (ES): calcd for $\mathrm{C}_{19} \mathrm{H}_{23} \mathrm{~F}_{6} \mathrm{O}_{8} \mathrm{PS}_{2} \mathrm{Na}[M+\mathrm{Na}]^{+}$: 611.0368; found: 611.0353.
Bis(trifluoromethylsulfonyl)silacyclobutene 21b. From $50 \mathrm{mg}$ (0.17 mmol) of alkyne 20b, and after flash chromatography of the residue using hexanes/ethyl acetate (99:1) as eluent gave compound $\mathbf{2 1 b}$ (80 $\mathrm{mg}, 81 \%)$ as a colorless oil; ${ }^{1} \mathrm{H}$ NMR (300 MHz, $\mathrm{CDCl}_{3}, 25{ }^{\circ} \mathrm{C}$ ): $\delta=7.42$ $\left(\mathrm{m}, 2 \mathrm{H}, 2 \mathrm{CH}^{\mathrm{Ar}}\right), 6.89\left(\mathrm{~m}, 2 \mathrm{H}, 2 \mathrm{CH}^{\mathrm{Ar}}\right), 3.84\left(\mathrm{~s}, 3 \mathrm{H}, \mathrm{OCH}_{3}\right)$, $3.32\left(\mathrm{~s}, 2 \mathrm{H}, \mathrm{CH}_{2}\right), 1.10\left(\mathrm{~m}, 21 \mathrm{H}, 3 \mathrm{CH}+6 \mathrm{CH}_{3}\right) ;{ }^{13} \mathrm{C} \mathrm{NMR}$ (75 MHz, $\left.\mathrm{CDCl}_{3}, 25^{\circ} \mathrm{C}\right): \delta=162.3(\mathrm{C}=\mathrm{C}-\mathrm{Si}), 160.7\left(C^{\mathrm{Ar}-\mathrm{q}_{-}}\right.$ $\left.\mathrm{OCH}_{3}\right), 149.0(\mathrm{C}=\mathrm{C}-\mathrm{Si}), 129.9\left(2 \mathrm{CH}^{\mathrm{Ar}}\right), 125.1\left(\mathrm{C}^{\mathrm{Ar}-\mathrm{q}}\right), 119.8$ $\left(\mathrm{q}, J_{C F}=331.1 \mathrm{~Hz}, 2 \mathrm{CF}_{3}\right), 113.5\left(2 \mathrm{CH}^{\mathrm{Ar}}\right), 90.1\left(\mathrm{CTf}_{2}\right), 55.2$ $\left(\mathrm{OCH}_{3}\right), 36.2\left(\mathrm{CH}_{2}\right), 18.4\left(6 \mathrm{CH}_{3}\right), 11.5(3 \mathrm{CH}) ;{ }^{19} \mathrm{~F}$ NMR (282 $\left.\mathrm{MHz}, \mathrm{CDCl}_{3}, 2{ }^{\circ} \mathrm{C}\right): \delta=-70.29\left(\mathrm{~s}, 6 \mathrm{~F}, 2 \mathrm{CF}_{3}\right)$; IR $\left(\mathrm{CHCl}_{3}\right): v=1614(\mathrm{C}=\mathrm{C}), 1379,1106(\mathrm{O}=\mathrm{S}=\mathrm{O}), 1203(\mathrm{C}-$ F) $\mathrm{cm}^{-1}$; HRMS (ES): calcd for $\mathrm{C}_{22} \mathrm{H}_{31} \mathrm{~F}_{6} \mathrm{NO}_{5} \mathrm{~S}_{2} \mathrm{Si}[M+$ $\left.\mathrm{NH}_{4}\right]^{+}$: 598.1546; found: 598.1566.

\section{Bis(trifluoromethylsulfonyl)bis(trifluoromethylsulfonyl} )clobutene 24c. From $20 \mathrm{mg}$ (0.047 mmol) of alkyne 22c, and after flash chromatography of the residue using hexanes/ethyl acetate (1:1) as eluent gave compound 24c (21 mg, 61\%) as a colorless solid; mp 153-155 ${ }^{\circ} \mathrm{C} ;{ }^{1} \mathrm{H}$ NMR (300 MHz, acetone- $\left.\mathrm{d}_{6}, 25^{\circ} \mathrm{C}\right): \delta=7.60\left(\mathrm{~m}, 2 \mathrm{H}, 2 \mathrm{CH}^{\mathrm{Ar}}\right.$ ), $6.96\left(\mathrm{~m}, 2 \mathrm{H}, 2 \mathrm{CH}^{\mathrm{Ar}}\right), 3.80\left(\mathrm{~s}, 3 \mathrm{H}, \mathrm{OCH}_{3}\right), 3.66\left(\mathrm{~s}, 2 \mathrm{H}, \mathrm{CH}_{2}\right)$, 3.51 (s, $2 \mathrm{H}, \mathrm{CH}_{2}$-cyclobutenyl). The signal of $\mathrm{CHTf}_{2}$ is not visible in the ${ }^{1} \mathrm{H}-\mathrm{RMN}$ spectrum because of its acidity; ${ }^{13} \mathrm{C}$ NMR (75 MHz, acetone-d $\left.6,25^{\circ} \mathrm{C}\right): \delta=161.3\left(C^{\mathrm{Ar}-\mathrm{q}}-\mathrm{OCH}_{3}\right)$, $156.4(C=\mathrm{C}), 130.5(\mathrm{C}=C), 130.2\left(2 \mathrm{CH}^{\mathrm{Ar}}\right), 123.5\left(\mathrm{C}^{\mathrm{Ar}-\mathrm{q}}\right)$, $122.6\left(\mathrm{q}, J_{C F}=327.7 \mathrm{~Hz}, 2 \mathrm{CF}_{3}\right), 120.9\left(\mathrm{q}, J_{C F}=331.1 \mathrm{~Hz}\right.$, 2. $\mathrm{CF}_{3}$-cyclobutenyl), $114.9 \quad\left(2 \mathrm{CH}^{\mathrm{Ar}}\right), \quad 86.9 \quad\left(\mathrm{CTf}_{2}-\right.$ cyclobutenyl), $61.2\left(\mathrm{CTf}_{2}\right), 55.8\left(\mathrm{OCH}_{3}\right), 37.5\left(\mathrm{CH}_{2-}\right.$ cyclobutenyl), $29.6\left(\mathrm{CH}_{2}\right)$. The signal of $\mathrm{CHTf}_{2}$ is visible in the ${ }^{13} \mathrm{C}$-RMN spectrum as a quaternary carbon rather than as a $\mathrm{CH}$ because of the deprotonation; ${ }^{19} \mathrm{~F}$ NMR $(282 \mathrm{MHz}$, acetone- $\left.\mathrm{d}_{6}, 25{ }^{\circ} \mathrm{C}\right): \delta=-72.04$ (s, 6F, 2 $\mathrm{CF}_{3}$-cyclobutenyl), -80.10 (s, 6F, 2CF $\mathrm{CF}_{3}$; IR (acetone): $v=1608(\mathrm{C}=\mathrm{C}), 1380$, $1099(\mathrm{O}=\mathrm{S}=\mathrm{O}), 1346,1042(\mathrm{O}=\mathrm{S}=\mathrm{O}), 1192(\mathrm{C}-\mathrm{F}) \mathrm{cm}^{-1}$; HRMS (ES): calcd for $\mathrm{C}_{17} \mathrm{H}_{16} \mathrm{~F}_{12} \mathrm{NO}_{9} \mathrm{~S}_{4}\left[M+\mathrm{NH}_{4}\right]^{+}$: 733.9511; found: 733.9513 .

Bis(trifluoromethylsulfonyl)thiocyclobutene 27a. From $15 \mathrm{mg}(0.027 \mathrm{mmol})$ of azide $\mathbf{2 6 a}$, and after flash chromatography of the residue using hexanes/ethyl acetate (8:2) as eluent gave compound 27a (17 mg, 85\%) as a yellow oil; ${ }^{1} \mathrm{H}$ NMR (700 MHz, $\mathrm{CDCl}_{3}, 2{ }^{\circ} \mathrm{C}$ ): $\delta=8.80$ (s, $1 \mathrm{H}, \mathrm{CH}$-triazolyl), 7.87 (m, 2H, 2CH ${ }^{\mathrm{Ar}}$ ), $7.76\left(\mathrm{~m}, 1 \mathrm{H}, \mathrm{CH}^{\mathrm{Ar}}\right)$, $7.63\left(\mathrm{~m}, 1 \mathrm{H}, \mathrm{CH}^{\mathrm{Ar}}\right), 7.54\left(\mathrm{~m}, 4 \mathrm{H}, 4 \mathrm{CH}^{\mathrm{Ar}}\right), 7.49(\mathrm{~m}, 1 \mathrm{H}$, $\mathrm{CH}^{\mathrm{Ar}}$ ), 3.68 (s, 2H, $\mathrm{CH}_{2}$ ); ${ }^{13} \mathrm{C}$ NMR (175 MHz, $\mathrm{CDCl}_{3}, 25$ $\left.{ }^{\circ} \mathrm{C}\right): \delta=165.9$ (C=C-S), 139.5 ( $\left.C^{\mathrm{Ar}-\mathrm{q}}-\mathrm{Tf}\right), 136.1(C=\mathrm{C}-\mathrm{S})$, $134.5\left(\mathrm{CH}^{\mathrm{Ar}}\right), 133.7\left(\mathrm{CH}^{\mathrm{Ar}}\right), 133.1\left(\mathrm{CH}^{\mathrm{Ar}}\right), 131.8\left(\mathrm{CH}^{\mathrm{Ar}}\right)$, $130.1\left(\mathrm{CH}^{\mathrm{Ar}}\right), 129.5\left(2 \mathrm{CH}^{\mathrm{Ar}}\right), 129.3\left(C^{\mathrm{Ar}-\mathrm{q}}\right), 128.1\left(2 \mathrm{CH}^{\mathrm{Ar}}\right)$, $127.4\left(C^{\mathrm{Ar}-\mathrm{q}}\right), 127.1\left(\mathrm{CH}^{\mathrm{Ar}}\right), 119.6\left(\mathrm{q}, J_{C F}=330.8 \mathrm{~Hz}, 2 \mathrm{CF}_{3}\right)$, $119.4\left(\mathrm{q}, J_{C F}=324.4 \mathrm{~Hz}, \mathrm{CF}_{3}\right), 116.7\left(\mathrm{C}^{\mathrm{Ar}-\mathrm{q}}\right), 88.0\left(\mathrm{CTf}_{2}\right)$, $35.5\left(\mathrm{CH}_{2}\right) ;{ }^{19} \mathrm{~F}$ NMR $\left(282 \mathrm{MHz}, \mathrm{CDCl}_{3}, 25^{\circ} \mathrm{C}\right): \delta=-70.44$ (s, 6F, 2CF 3$),-78.50\left(\mathrm{~s}, 3 \mathrm{~F}, \mathrm{CF}_{3}\right)$; IR $\left(\mathrm{CHCl}_{3}\right): v=1385$, $1104(\mathrm{O}=\mathrm{S}=\mathrm{O}), 1214(\mathrm{C}-\mathrm{F}) \mathrm{cm}^{-1}$; HRMS (ES): calcd for $\mathrm{C}_{21} \mathrm{H}_{16} \mathrm{~F}_{9} \mathrm{~N}_{4} \mathrm{O}_{6} \mathrm{~S}_{4}\left[M+\mathrm{NH}_{4}\right]^{+}$: 718.9803; found: 718.9797 .

General procedure for the uncatalyzed reaction of heteroatom-substituted alkynes $10 \mathrm{~m}-\mathrm{t}$ and pyridinium salt 2. Synthesis of cyclobutenones 12m-t. 2-(2Fluoropyridin-1-ium-1-yl)-1,1-

bis[(trifluoromethyl)sulfonyl]ethan-1-ide 2 (0.2 mmol) was 
added at room temperature to a solution of the appropriate ynamine $10 \mathrm{~m}-\mathbf{t}(0.2 \mathrm{mmol})$ in acetonitrile $(4 \mathrm{~mL})$. The reaction was stirred at room temperature until disappearance of the starting material (TLC). Saturated potassium carbonate $(2 \mathrm{~mL})$ was added and the mixture was stirred for 10 minutes, before being partitioned between ethyl acetate and water. The organic extract was washed with brine, dried $\left(\mathrm{MgSO}_{4}\right)$, and concentrated under reduced pressure. Chromatography of the residue eluting with hexanes/ethyl acetate mixtures gave analytically pure compounds. Spectroscopic and analytical data for adducts $\mathbf{1 2 m - t}$ follow.

Aminocyclobutenone 12p. From $20 \mathrm{mg}(0.07 \mathrm{mmol})$ of alkyne 10p, and after flash chromatography of the residue using hexanes/ethyl acetate (85:15) as eluent gave compound 12p (20 mg, 88\%) as a colorless solid; mp 154$156{ }^{\circ} \mathrm{C} ;{ }^{1} \mathrm{H}$ NMR (300 MHz, $\mathrm{C}_{6} \mathrm{D}_{6}, 25{ }^{\circ} \mathrm{C}$ ): $\delta=7.96$ (d, $2 \mathrm{H}$, $\left.J=7.7 \mathrm{~Hz}, 2 \mathrm{CH}^{\mathrm{Ar}}\right), 7.41\left(\mathrm{~d}, 2 \mathrm{H}, J=8.0 \mathrm{~Hz}, 2 \mathrm{CH}^{\mathrm{Ar}}\right), 7.28$ $\left(\mathrm{m}, 2 \mathrm{H}, 2 \mathrm{CH}^{\mathrm{Ar}}\right), 7.21\left(\mathrm{~m}, 2 \mathrm{H}, 2 \mathrm{CH}^{\mathrm{Ar}}\right), 6.95\left(\mathrm{~m}, 2 \mathrm{H}, 2 \mathrm{CH}^{\mathrm{Ar}}\right)$, $6.38\left(\mathrm{~m}, 2 \mathrm{H}, 2 \mathrm{CH}^{\mathrm{Ar}}\right), 3.11\left(\mathrm{~s}, 2 \mathrm{H}, \mathrm{CH}_{2}\right) .3 .07$ (s, 3H, $\left.\mathrm{OCH}_{3}\right)$; ${ }^{13} \mathrm{C}$ NMR (75 MHz, $\left.\mathrm{C}_{6} \mathrm{D}_{6}, 25{ }^{\circ} \mathrm{C}\right): \delta=183.8(\mathrm{C}=\mathrm{O}), 162.5$ $\left(C^{\mathrm{Ar}-\mathrm{q}}-\mathrm{OCH}_{3}\right), \quad 157.7 \quad(\mathrm{C}=\mathrm{C}-\mathrm{N}), \quad 139.0 \quad\left(2 C^{\mathrm{Ar}-\mathrm{q}}\right), \quad 132.9$ $\left(2 \mathrm{CH}^{\mathrm{Ar}}\right), 128.7(\mathrm{C}=\mathrm{C}-\mathrm{N}), 126.5\left(2 \mathrm{CH}^{\mathrm{Ar}}\right), 124.5\left(2 \mathrm{C}^{\mathrm{Ar}-\mathrm{q}}\right)$, $123.9\left(C^{\mathrm{Ar}-\mathrm{q}}\right), 121.3\left(2 \mathrm{CH}^{\mathrm{Ar}}\right), 120.7\left(2 \mathrm{CH}^{\mathrm{Ar}}\right), 114.3\left(2 \mathrm{CH}^{\mathrm{Ar}}\right)$, $112.5\left(2 \mathrm{CH}^{\mathrm{Ar}}\right), 54.8\left(\mathrm{OCH}_{3}\right), 45.5\left(\mathrm{CH}_{2}\right)$; IR $\left(\mathrm{CH}_{2} \mathrm{Cl}_{2}\right): v=$ $1760(\mathrm{C}=\mathrm{O}), 1602(\mathrm{C}=\mathrm{C}), 1262(\mathrm{C}-\mathrm{O}) \mathrm{cm}^{-1}$; HRMS (ES): calcd for $\mathrm{C}_{23} \mathrm{H}_{18} \mathrm{NO}[M+\mathrm{H}]^{+}$: 340.1332; found: 340.1324.

\section{Acknowledgements}

Support for this work by the MINECO and FEDER (Projects CTQ2015-65060-C2-1-P and CTQ2015-65060-C2-2-P). C. L.-M. thanks MINECO for a predoctoral grant. We thank Prof. H. Yanai for the kind donation of bis(trifluoromethylsulfonyl)methane. We are grateful to Dr. M. R. Torres for X-ray analysis.

\section{References}

[1] a) Y. Aoyagi, A. Yamazaki, R. Kato, F. Tobe, H. Fukaya, T. Nishikawa, A. Nakahashi, N. Miura, K. Monde, K. Takeya, Tetrahedron Lett. 2011, 52, 1851; b) T. Seiser, T. Saget, D. N. Tran, N. Cramer, Angew. Chem. 2011, 123, 7884; Angew. Chem. Int. Ed. 2011, 50,7740; c) N. Hoffmann, Chem. Rev. 2008, 108, 1052; d) V. M. Dembitsky, J. Nat. Med. 2008, 62, 1; e) J.-J. Tan, C.-H. Tan, Y.-Q. Wang, S.-H. Jiang, D.-Y. Zhu, Helv. Chim. Acta 2006, 89, 117; f) E. Lee-Ruff, G. Mladenova, Chem. Rev. 2003, 103, 1449.

[2] For selected recent references, see: a) K.-i. Takao, R. Nemoto, K. Mori, A. Namba, K. Yoshida, A. Ogura, Chem. Eur. J. 2017, 23, 3828; b) M. Eisold, A. N. Baumann, G. M. Kiefl, S. T. Emmerling, D. Didier, Chem. Eur. J. 2017, 23, 1634; c) T. Kurohara, M. Shibuya, Y. Yamamoto, Adv. Synth. Catal. 2017, 359, doi:10.1002/adsc.201601297; d) S. Blumberg, S. F. Martin, Org. Lett. 2017, 19, 790; e) C.-Z. Zhong, P.-T. Tung, T.-H. Chao, M.-C. P. Yeh, J. Org. Chem. 2017, 82, 481; f) X. Mao, P. Song, Y. Hao, Z. Sun, X. Hu, Adv. Synth. Catal. 2016, 358, 3719; g) P.-h. Chen, G. Dong, Chem. Eur. J. 2016, 22, 18290; h) S. Reboredo, R. M.
Girón, S. Filippone, T. Mikie, T. Sakurai, S. Seki, N. Martín, Chem. Eur. J. 2016, 22, 13627; i) W. Zhang, J. M. Ready, J. Am. Chem. Soc. 2016, 138, 10684; j) M. Guisán-Ceinos, A. Parra, V. Martín-Heras, M. Tortosa, Angew. Chem. 2016, 128, 7803; Angew. Chem. Int. Ed. 2016, 55, 6969; k) M. Eisold, G. M. Kiefl, D. Didier, Org. Lett. 2016, 18, 3022; l) T. Matsuda, T. Matsumoto, Org. Biomol. Chem. 2016, 14, 5023; m) Y. Qiu, B. Yang, C. Zhu, J.-E. Bäckvall, Angew. Chem. 2016, 128, 6630; Angew. Chem. Int. Ed. 2016, 55, 6520; n) T. Kang, S. Ge, L. Lin, Y. Lu, X. Liu, X. Feng, Angew. Chem. 2016, 128, 5631; Angew. Chem. Int. Ed. 2016, 55, 5541; o) B. Ranieri, C. Obradors, M. Mato, A. M. Echavarren, Org. Lett. 2016, 18, 1614; p) P. Song, Q. Li, C. Wang, W. Wu, X. Mao, J. Wang, X. Hu, Adv. Synth. Catal. 2016, 358, 1208; q) S. A. Ruider, E. M. Carreira, Org. Lett. 2016, 18, 220; r) S. Blouin, V. Gandon, G. Blond, J. Suffert, Angew. Chem. 2016, 128, 7324; Angew. Chem. Int. Ed. 2016, 55, 7208; s) M. Eisold, D. Didier, Angew. Chem. 2015, 127, 16112; Angew. Chem. Int. Ed. 2015, 54, 15884; t) S. Yang, W. Yuan, Q. Xu, M. Shi, Chem. Eur. J. 2015, 21, 15964; u) M. J. Ralph, D. C. Harrowven, S. Gaulier, S. Ng, K. I. BookerMilburn, Angew. Chem. 2015, 127, 1547; Angew. Chem. Int. Ed. 2015, 54, 1527; v) N. Arichi, K. Yamada, Y. Yamaoka, K. Takasu, J. Am. Chem. Soc. 2015, 137, 9579; w) C. Souris, A. Misale, Y. Chen, M. Luparia, N. Maulide, Org. Lett. 2015, 17, 4486; x) B. D. Robertson, R. E. M. Brooner, R. A. Widenhoefer, Chem. Eur. J. 2015, 21, 5714; y) X.-N. Wang, E. H. Krenske, R. C. Johnston, K. N. Houk, R. P. Hsung, J. Am. Chem. Soc. 2014, 136, 9802; z) R. H. Grubbs, J. Hartung, Angew. Chem. 2014, 126, 3966; Angew. Chem. Int. Ed. 2014, 53, 3885.

[3]B. Alcaide, P. Almendros, I. Fernández, C. Lázaro-Milla, Chem. Commun. 2015, 51, 3395.

[4] For a recent review on the peculiar properties of fluoroorganic molecules, see: E. P. Gillis, K. J. Eastman, M. D. Hill, D. J. Donelly, N. A. Meanwell, J. Med. Chem. 2015, 58, 8315.

[5] For selected strategies to prepare triflones, see: a) $\mathrm{H}$. Yanai, R. Takahashi, Y. Takahashi, A. Kotani, H. Hakamata, T. Matsumoto, Chem. Eur. J. 2017, 23, DOI: 10.1002/chem.201700515; b) L.-N. Huan, F.-L. Qing, Y. Huang, Y. Sumii, X.-H. Xu, Org. Biomol. Chem. 2016, 14, 8443; c) Z. Huang, C. Wang, E. Tokunaga, Y. Sumii, N. Shibata, Org. Lett. 2015, 17, 5610; d) B. Alcaide, P. Almendros, C. Lázaro-Milla, Chem. Commun. 2015, 51, 6992; e) H. Kawai, Y. Sugita, E. Tokunaga, H. Sato, M. Shiro, N. Shibata, ChemistryOpen 2014, 3, 14; f) H. Yanai, Y. Takahashi, H. Fukaya, Y. Dobashi, T. Matsumoto, Chem. Commun. 2013, 49, 10091; g) H. Kawai, Y. Sugita, E. Tokunaga, N. Shibata, Eur. J. Org. Chem. 2012, 1295; h) H. Yanai, H. Ogura, H. Fukaya, A. Kotani, F. Kusu, T. Taguchi, Chem. Eur. J. 2011, 17, 11747; i) H. Yanai, M. Fujita, T. Taguchi, Chem. Commun. 2011, 47, 7245.

[6]CCDC 1060047 contains the supplementary crystallographic data for compound $\mathbf{3 d}$ in this paper. These data can be obtained free of charge from The 
Cambridge Crystallographic Data Centre via www.ccdc.cam.ac.uk/data_request/cif.

[7] For selected reviews, see: a) D. H. Ortgies, A. Hassanpour, F. Chen, S. Woo, P. Forgione, Eur. J. Org. Chem. 2016, 408; b) R. E. Click, J. Reprod. Develop. 2013, 49, 1282; c) T. Zeng, C.-L. Zhang, X.-L. Zhao, Crit. Rev. Food. Sci. 2013, 53, 215; d) H. R. Vasanthi, S. Mukherjee, D. K. Das, Mini-Rev. Med. Chem. 2009, 9, 749; e) M. S. Butt, M. T. Sultan, M. S. Butt, J. Iqbal, Crit. Rev. Food. Sci. 2009, 49, 538; f) R. Naithani, C. L. Huma, L. E. Holland, D. Shukla, D. L. McCormick, R. G. Mehta, R. M. Moriarty, Mini-Rev. Med. Chem. 2008, 8, 1106; g) M. Corzo-Martínez, N. Corzo, M. Villamiel, Trends Food Sci. Tech. 2007, 18, 609; h) D. Witt, R. Klajn, P. Barski, B. A. Grzybowski, Curr. Org. Chem. 2004, 8, 1763.

[8] Organoselenium compounds are important reagents, synthons, and catalysts: a) Q.-S. Li, D.-M. Wu, B.-C. Zhu, Y.-G. Wang, Mini-Rev.

Med.

Chem. 2013, 13, 854; b) T. Wirth, Organoselenium Chemistry: Synthesis and Reactions, WileyVCH: Weinheim, 2011; c) I. P. Beletskaya, V. P. Ananikov, Chem. Rev. 2011, 111, 1596; d) B. Godoi, R. F. Schumacher, G. Zeni, Chem. Rev. 2011, 111, 2937. Organoselenium derivatives display antioxidant, antibacterial, antitumor, antihypertensive, enzyme modulator, antiviral, and antithyroid properties: e) J.-Y. Yang, C.-S. Xu, Pharmacol. Rep. 2009, 61, 288; f) G. Roy, G. Mugesh, Chem. Biodivers. 2008, 5, 414; g) C. Sanmartin, D. Plano, J. A. Palop, Mini-Rev. Med. Chem. 2008, 8,1020; h) C. W. Nogueira, G. Zeni, J. B. T. Rocha, Chem. Rev. 2004, 104, 6255; i) M. SorianoGarcía, Curr. Med. Chem. 2004, 11, 1657; j) T. G. Chasteen, R. Bentley, Chem. Rev. 2003, 103, 1; k) M. Birringer, S. Pilawa, L. Flohe, Nat. Prod. Rep. 2002, 19, 693.

[9] Strong effects of the substitution pattern have been observed, because related ynamides afforded cyclobutenols rather than cyclobutenones: B. Alcaide, P. Almendros, C. Lázaro-Milla, Chem. Eur. J. 2016, 22, 8998.

[10] Cyclobutenones are relevant synthetic intermediates. For selected reviews, see: a) T. Xu, A. Dermenci, G. Dong, Top. Curr. Chem. 2014, 346, 233; b) D. Bellus, B. Ernst, Angew. Chem. 1988, 100, 820; Angew. Chem. Int. Ed. 1988, 27, 797. For a recent contribution, see: c) M. Pareek, R. B. Sunoj, Org. Lett. 2016, 18, 5932.

[11] For dynamic chirality control studies of phosphinyl cyclobutenes, see: S. Ito, M. Nanko, T. Shinozaki, M. Kojima, K. Aikawa, K. Mikami, Chem. Asian J. 2016, $11,823$.

[12] Fort he synthesis and characterization of $\mathrm{Tf}_{2} \mathrm{C}$-based zwitterions containing a carbanion moiety, see: H. Yanai, T. Yoshino, M. Fujita, H. Fukaya, A. Kotani, F. Kusu, T. Taguchi, Angew. Chem. 2013, 125, 1600; Angew. Chem. Int. Ed. 2013, 52, 1560.

[13] CCDC 1438231 contains the supplementary crystallographic data for compound 24c in this paper. These data can be obtained free of charge from The Cambridge Crystallographic Data Centre via www.ccdc.cam.ac.uk/data_request/cif.

[14] Experimental procedures as well as full spectroscopic and analytical data for compounds not included in this Experimental Section are described in the Supporting Information. It contains compound characterization data, experimental procedures, and copies of NMR spectra for all new compounds. 


\section{FULL PAPER}

Regioselective Synthesis of HeteroatomFunctionalized Cyclobutene-triflones and Cyclobutenones

Adv. Synth. Catal. 2017, 359, Page - Page

Benito Alcaide,* Pedro Almendros,* and Carlos Lázaro-Milla

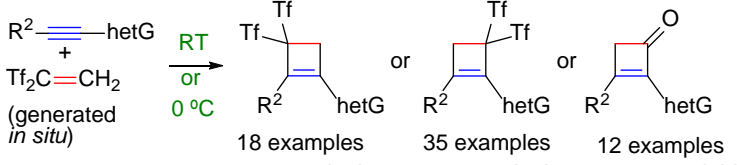

$$
\begin{aligned}
& \text { up to quantitative up to quantitative up to } 86 \% \text { yield } \\
& \text { yield } \\
& \text { yield }
\end{aligned}
$$

Alcaide, B.; Almendros, P.; Lázaro-Milla, C.

Título: Regioselective Synthesis of Heteroatom-Functionalized Cyclobutene-Triflones and Cyclobutenones

Revista: Adv. Synth. Catal. 2017, 359, 2630-2639; DOI: 10.1002/adsc.201700492 